\title{
Sox21 Promotes Hippocampal Adult Neurogenesis via the Transcriptional Repression of the Hes5 Gene
}

\author{
Satoru Matsuda, ${ }^{1,2 *}$ Ken-ichiro Kuwako, ${ }^{1 \star}$ Hirotaka James Okano, ${ }^{1}$ Shuichi Tsutsumi, ${ }^{5}$ Hiroyuki Aburatani, ${ }^{5}$ \\ Yumiko Saga,${ }^{6}$ Yumi Matsuzaki, ${ }^{1}$ Akinori Akaike, ${ }^{3}$ Hachiro Sugimoto, ${ }^{2,4}$ and Hideyuki Okano ${ }^{1}$ \\ ${ }^{1}$ Department of Physiology, Keio University School of Medicine, Shinjuku-ku, Tokyo 160-8582, Japan, Departments of ${ }^{2}$ Neuroscience for Drug Discovery \\ and ${ }^{3}$ Pharmacology, Graduate School of Pharmaceutical Sciences and ${ }^{4}$ World-Leading Drug Discovery Research Center, Kyoto University, Sakyo-ku, Kyoto \\ 606-8501, Japan, ${ }^{5}$ Genome Science Division, Research Center for Advanced Science and Technology, The University of Tokyo, Meguro-ku, Tokyo 153-8904, \\ Japan, and ${ }^{\circ}$ Division of Mammalian Development, National Institute of Genetics, Mishima, Shizuoka 411-8540, Japan
}

Despite the importance of the production of new neurons in the adult hippocampus, the transcription network governing this process remains poorly understood. The High Mobility Group (HMG)-box transcription factor, Sox2, and the cell surface activated transcriptional regulator, Notch, play important roles in CNS stem cells. Here, we demonstrate that another member of the SoxB (Sox1/Sox2/Sox3) transcription factor family, Sox21, is also a critical regulator of adult neurogenesis in mouse hippocampus. Loss of Sox 21 impaired transition of progenitor cells from type $2 a$ to type $2 b$, thereby reducing subsequent production of new neurons in the adult dentate gyrus. Analysis of the Sox 21 binding sites in neural stem/progenitor cells indicated that the Notch-responsive gene, Hes5, was a target of Sox 21 . Sox 21 repressed Hes 5 gene expression at the transcriptional level. Simultaneous overexpression of Hes 5 and Sox 21 revealed that Hes 5 was a downstream effector of Sox 21 at the point where the Notch and Sox pathways intersect to control the number of neurons in the adult hippocampus. Therefore, Sox 21 controls hippocampal adult neurogenesis via transcriptional repression of the Hes5 gene.

\section{Introduction}

Endogenous neural stem/progenitor cells (NS/PCs) continuously give rise to neurons in the adult dentate gyrus (DG) of the hippocampus (Gage 2000; Duan et al., 2008). The "adult neurogenesis" phenomenon comprises several stages, which are strictly regulated by distinct mechanisms (Kempermann et al., 2004); however, the transcriptional network that governs adult neurogenesis remains largely unknown. The SRY-box containing gene (Sox) family, which encodes High Mobility Group (HMG)-box transcription factors, is essential for CNS development and for maintaining adult neurogenesis (Lefebvre et al., 2007). Among the members of this family, Sox1/Sox2/Sox3 (SoxB1) is reported to suppress neuronal differentiation by maintaining NS/PCs in an undifferentiated state (Kan et al., 2004; Bani-Yaghoub et al.,

Received Nov. 21, 2011; revised June 21, 2012; accepted July 17, 2012.

Author contributions: S.M., K.-i.K., H.J.O., H.A., A.A., H.S., and H.O. designed research; S.M., K.-i.K., and S.T. performed research; Y.S. and Y.M. contributed unpublished reagents/analytic tools; S.M. and S.T. analyzed data; S.M., K.-i.K., S.T., and H.O. wrote the paper.

This work was supported by grants from the Japan Society for the Promotion of Science (S.M.), the Japanese Ministry of Education, Culture, Sports, Science and Technology (H.O.), Funding Program for World-leading Innovative R\&D on Science and Technology (H.O.), Solution-Oriented Research for Science and Technology (SORST) of the Japan Science and Technology Agency (H.O.), and the Bridgestone Corporation. The funders had no role in study design, data collection, data analysis, the decision to publish, or preparation of this manuscript. We are grateful to Dr. F. H. Gage (Salk Institute for Biological Studies) for providing AHP cells. We also thank H. Naka-Kaneda (RIKEN Yokohama Institute), C. Hara-Miyauchi (Tokyo Medical and Dental University), F. Ozawa, Y. Imaizumi, S. Suzuki (Keio University), and N. Kaneko (Nagoya (ity University) for their expert technical assistance, and S. Shibata, K. Kakumoto (Keio University), T. Sunabori (Juntendo University), S. Fukami (Nara Medical University), K. Sawamoto (Nagoya City University), and other members of the 0 kano laboratory for helpful discussions and encouragement.

*S.M. and K.-i.K. contributed equally to this work.

Correspondence should be addressed to Hideyuki 0kano at the above address. E-mail: hidokano@a2.keio.jp.

DOI:10.1523/JNEUROSCI.5803-11.2012

Copyright $\odot 2012$ the authors $\quad 0270-6474 / 12 / 3212543-15 \$ 15.00 / 0$
2006; Wang et al., 2006). In addition, Sox2-positive cells in the adult DG are thought to be NS/PCs, which contribute to the production of new neurons (Suh et al., 2007). Our previous immunohistochemical study using antibodies against SoxB1 and Sox 21 demonstrated restricted expression of Sox 21 in neurogenic regions in both embryonic and adult mouse brains (Tada et al., 2007). The neurogenic potential of Sox 21 was indicated in the chick embryonic spinal cord, where it counteracts the effects of SoxB1 (Sandberg et al., 2005). Thus, Sox 1 could conceivably also be involved in the regulation of adult neurogenesis in mice.

The Notch signaling pathway is also important for the regulation of CNS development, as it controls the proliferation and cell fate decisions of NS/PCs via downstream effectors such as Hes and Hey (Lewis 1996; Lathia et al., 2008). Recently, the Notch pathway was identified as a key regulator of the neurogenic niche in adult brains (Breunig et al., 2007; Imayoshi et al., 2010; Lugert et al., 2010). Thus, understanding the modulation of Notch signaling during adult neurogenesis may facilitate better understanding of the entire mechanism of adult neurogenesis.

The present study used Sox21-deficient $\left(\right.$ Sox $\left.21^{-/-}\right)$mice to examine the in vivo function of the transcription factor Sox21 during hippocampal adult neurogenesis. Moreover, as a first step to unveiling the gene network downstream of Sox21, the target genes of Sox 21 were screened using chromatin immunoprecipitation (ChIP) sequencing. Sox 21 transcriptionally repressed the expression of Hes5, a known target of Notch signaling. By repressing Hes5, Sox 21 contributed to the cancelation of the undifferentiated status of NS/PCs and promoted neurogenesis. Thus, this study demonstrates a pivotal role for Sox 21 in the regulation of adult neurogenesis. 


\section{Materials and Methods}

Animals. All animal care and treatment procedures were performed in accordance with institutional guidelines approved by the Experimental Animal Care Committee of the Keio University School of Medicine. Animals were housed in a room with a $12 \mathrm{~h}$ light/dark cycle and fed ad libitum. Sox $21^{-1-}$ mice were established as described previously (Kiso et al., 2009) and maintained on a C57BL6/J background. The following primers were used to determine genotypes: wild-type alleles, $5^{\prime}$-CTCA TCCTTCCTCCCTCCCG-3' and 5'-CCAAGCCAGCGGACTCAGAG AC- $3^{\prime}$; mutant alleles, $5^{\prime}$-CGATCACATGGTCCTGCTGGAGT- ${ }^{\prime}$ ' and 5'-CCAAGCCAGCGGACTCAGAGAC-3' (same primer as for the wildtype alleles). Hes5-NLSlacZ knock-in mouse brains were kindly provided by Dr. R. Kageyama (Kyoto University, Kyoto, Japan) (Imayoshi et al., 2010). Animals of both sexes were used in this study.

BrdU treatment. Proliferating cells in the DG were labeled by short- or long-term administration of BrdU as previously described (Sakaguchi et al., 2006). Briefly, for long-term labeling, mice were administered 1 $\mathrm{mg} / \mathrm{ml}$ of BrdU via the drinking water for 2 weeks and killed 4 weeks after the last day of administration. For short-term labeling, mice were injected intraperitoneally with $50 \mathrm{mg} / \mathrm{kg}$ of BrdU (Sigma-Aldrich) three times at $3 \mathrm{~h}$ intervals and killed $3 \mathrm{~h}$ after the last injection. To analyze embryonic neurogenesis, mice were injected intraperitoneally with 200 $\mathrm{mg} / \mathrm{kg}$ of BrdU at embryonic day 15.5 (E15.5) and killed $1.5 \mathrm{~h}$ after the injection or on postnatal day 7 (P7).

Histological analysis. Animals were perfused with $4 \%(\mathrm{w} / \mathrm{v})$ paraformaldehyde (Nakalai Tesque) and the brains dissected, postfixed in $4 \%$ paraformaldehyde at $4^{\circ} \mathrm{C}$ overnight, and sliced into $50 \mu \mathrm{m}$ sections using a vibratome (Leica). To detect $\mathrm{BrdU}$ incorporation, tissue sections were pretreated with $1 \mathrm{~N} \mathrm{HCl}$ at $37^{\circ} \mathrm{C}$ for $30 \mathrm{~min}$. To detect Ascl1-positive cells, tissue sections were pretreated with ice-cold acetone for $1 \mathrm{~min}$. The sections were preblocked with Tris- $\mathrm{NaCl}$ blocking buffer containing $0.3 \%$ Triton X-100 for $1 \mathrm{~h}$ at room temperature (PerkinElmer) and incubated with primary antibodies at $4^{\circ} \mathrm{C}$ overnight followed by Alexa Fluor-, HRP-, or biotin-conjugated secondary antibodies for $90 \mathrm{~min}$ at room temperature. The Vectastain Elite ABC kit (Vector Laboratories) and/or Tyramide Signal Amplification (TSA)-Red or TSA-Green (PerkinElmer) were used to visualize HRP- and biotin-conjugated antibodies. Nuclei were counterstained with Hoechst $33258(10 \mu \mathrm{g} / \mathrm{ml}$; Sigma-Aldrich, B2883) for $10 \mathrm{~min}$ at room temperature. To visualize the structures of the hippocampus and cerebellum at P7, sections were stained with $0.2 \%$ cresyl violet (Nissl staining).

Antibodies for immunohistochemistry. The following primary antibodies were used in this study: anti- $\beta$ III-tubulin (rabbit IgG; Covance, PRB435P; 1:500), anti-glial fibrillary acidic protein (GFAP; rabbit IgG; DAKO, Z0334; 1:500), anti-GFAP (mouse IgG; Sigma-Aldrich, G3893; 1:200), anti-fatty acid binding protein 7 (FABP7; rabbit IgG; Millipore, AB9558; 1:250), anti-Sox21 (goat IgG; R \& D Systems, AF3538; 1:100), anti-Sox2 (rabbit IgG; Millipore, AB5603; 1:200), anti-Sox1/(2)/3 (rabbit IgG; gift from Dr. H. Kondoh, Osaka University, Osaka, Japan; 1:5000) (Tanaka et al., 2004), anti-Nestin (mouse IgG; BD Biosciences/PharMingen, 556309; 1:200), anti-doublecortin (DCX; goat IgG; Santa Cruz Biotechnology, sc-8066; 1:100), anti- DCX (rabbit IgG; Abcam, ab18723; 1:100), anti-polysialylated neuronal cell adhesion molecule (PSANCAM; mouse IgM $\kappa$; gift from Dr. T. Seki, Tokyo Medical University, Tokyo, Japan; 1:200), anti-NeuN (mouse IgG; Millipore, MAB377; $1: 100$ ), anti-S100 $\beta$ (mouse IgG; Sigma-Aldrich, S2532; 1:200), antiglutathione $S$-transferase $\pi$ (mouse IgG; BD Biosciences/PharMingen, 610719; 1:200), anti-Brn2 (Santa Cruz Biotechnology, sc-6029; 1:100), anti-Tbr1 (rabbit IgG; gift from Dr. R. F. Hevner, University of Washington, Seattle, WA; 1:10,000), anti-Ascl1 (mouse IgG; BD Biosciences/ PharMingen, 556604; 1:100), anti-Tbr2 (rabbit IgG; Abcam, ab23345; 1:200), anti-Ki67 (rabbit IgG; Abcam, ab15580; 1:3000), anti-GFP (rabbit IgG, Medical and Biological Laboratories, 598, 1:500; goat IgG, Rockland, 600-101-215, 1:500), anti-BrdU (rat IgG; Abcam, ab6326; 1:100), anti-lacZ (rabbit IgG, Cappel, 55976, 1:500; goat IgG, Cappel, 56028, 1:500), and normal IgG (rabbit IgG, Millipore, DAM1421465; goat IgG, Santa Cruz Biotechnology, sc-2028). Immunoreactivity was visualized using Alexa Fluor-conjugated secondary antibodies (Invitrogen; 1:250) and HRP- or biotin-conjugated secondary antibodies (Jackson ImmunoResearch; 1:250).

Cell culture. The adult rat hippocampal progenitor (AHP) cell line was established as described previously (Palmer et al., 1997). Cultures were maintained on poly-L-ornithine-coated (Sigma-Aldrich) and laminincoated (Sigma-Aldrich) dishes in DMEM/F-12 (1:1; Invitrogen) supplemented with N2 (Invitrogen) and $40 \mathrm{ng} / \mathrm{ml}$ recombinant human FGF-2 from Escherichia coli (PeproTech).

Retrovirus preparation. To prepare the retrovirus vectors, a pMYs-Sox21IRES-Egfp or pMYs- $3 \times$ flag-Hes5-IRES-Eg $f p$ plasmid was constructed by inserting the mouse Sox21 (NC_000080.5) or Hes5 (NC_000070.5) fragments into a pMYs-IG backbone (Kitamura et al., 2003). The open reading frames of Sox 21 and Hes5 were PCR amplified from the pcDNA/Sox 21 vector (Ohba et al., 2004) and from a cDNA library of neurospheres derived from embryonic stem cells, respectively. The following primers were used for subcloning the cDNA: Sox21, 5' -ccgggatccgccaccATGTCCAAGCCTGTGGAC CACGTCAAGC- $3^{\prime}$ and $5^{\prime}$-ccgctcgagTCATAGCGCGGCAGCGTAGGCCG CGGGGTAG-3'; Hes5, 5' -ccggaattcgccaccATGGCCCCAAGTACCGTG-3' and $5^{\prime}$-ccgctcgagTCACCAGGGCCGCCAGAGG-3'. Underlined letters in the sequences represent the restriction sites used for subcloning. The gccacc sequence is the Kozak sequence. The pMY-Sox21-2A-3×flag-Hes5-IRES$E g f p$ construct was generated by subcloning the Sox 21 fragment without a stop codon (primer sequences: $5^{\prime}$-ccggtcgacgccaccATGTCCAAGCCTGTG GACCACGTCAAGC-3' and 5'-ccgggatccTAGCGCGGCAGCGTAGGCC GCGGGGTAG-3'). The 2A sequence was a gift from Dr. T. Maeda (Central Institute for Experimental Animals, Kanagawa, Japan). The $3 \times$ FLAG tag sequence was derived from the $3 \times$ FLAG-CMV-7 plasmid (Sigma-Aldrich).

Retrovirus was produced by transient transfection of Plat-E cells (Kitamura et al., 2003) with retrovirus constructs using GeneJuice (Novagen) according to the manufacturer's instructions. The medium was changed $24 \mathrm{~h}$ after transfection, and the supernatants were harvested $72 \mathrm{~h}$ after transfection. To ensure efficient in vitro and in vivo infection, the viral particles were concentrated by centrifugation at $8000 \mathrm{rpm}$ for $16 \mathrm{~h}$ and resuspended in HBSS (Invitrogen).

In vivo viral infection. A retrovirus suspension $(1 \mu \mathrm{l})$ was stereotactically injected into the DG of the hippocampus (coordinates relative to bregma, 2.0 $\mathrm{mm}$ posterior, $1.5 \mathrm{~mm}$ lateral, and $2.3 \mathrm{~mm}$ ventral from the skull) of anesthetized 6-week-old C57BL6 wild-type mice (purchased from Nihon SLC). After 3, 21, or $28 \mathrm{~d}$, the mice were transcardially perfused with PBS and $4 \%$ PFA, and the brains were subjected to immunohistochemical analyses. To analyze the phenotype of the infected cells, at least $20 \mathrm{GFP}$-expressing cells in the subgranular zone (SGZ) and granule cell layer (GCL) were observed from each individual mouse, and the percentage GFP $+/ \mathrm{NeuN}+$ cells among the total GFP + cell population was calculated.

ChIP-quantitative PCR and ChIP sequencing. ChIP was performed as described previously (Kaneshiro et al., 2007; Wakabayashi et al., 2009) with some modifications. Briefly, for ChIP-quantitative PCR (qPCR), AHP cells were harvested, and genomic DNA was cross-linked using $1 \%$ formaldehyde overnight at $4^{\circ} \mathrm{C}$. Cross-linking was terminated by the addition of $125 \mathrm{~mm}$ glycine, and the chromatin was sonicated using an Ultrasonic Disrupter (Tomy Digital Biology; 10\% duty, output level 1) for $10 \mathrm{~h}$ at $4^{\circ} \mathrm{C}$. The sonicated chromatin was incubated with anti-Sox 21 or anti-Sox2 antibodies coupled to magnetic DynaBeads (Active Motif) overnight at $4^{\circ} \mathrm{C}$. The beads were washed five times with $\mathrm{NaCl}$ buffer, once with LiCl buffer (Millipore, 20-156), and twice with Tris-EDTA buffer. After washing, the DNA bound to the beads was eluted in elution buffer ( $1 \% \mathrm{SDS}, 0.1 \mathrm{M} \mathrm{NaHCO}$ ) overnight at $65^{\circ} \mathrm{C}$ to remove cross-links and desalted using the QIAquick PCR purification kit (Qiagen). ChIP samples were analyzed by real-time PCR using SYBR Premix Ex Taq (TaKaRa Biotechnology, \#R0041B) and the following primers: mouse Hes5 proximal promoter, 5'-CTGGGAAAAGGCAGCATATTGAG-3' and 5'-ACGCTAAATTGCCTGTGAATTG-3'; mouse Hes 5 distal promoter, 5'-GATCCTCTGAAAGGTACAGGAAGG-3' and 5'-CTCACA CCATCCGACTTGAGTA-3'; rat Hes 5 proximal promoter, $5^{\prime}$-GAAGGG AAGAAGGGAGAGAAGG-3' and $5^{\prime}$-ACGCTAAATTGCCTGTGAAT TG-3'; and rat Hes 5 distal promoter, $5^{\prime}$-GATCCTCTGAAAGGTACAGG AAGG- 3 ' and $5^{\prime}$-CTCACACCATCCGACTTGAGTA- 3 '.

For ChIP sequencing, neurosphere cultures from the forebrains of E11.5 mouse embryos were used as the cell source. The DNA was purified 


\section{Sox21/Ki67/Hoechst Sox21/BrdU/Hoechst Sox21/ßIII-tub/Hoechst}

\section{Sox21/ßIII-tub/Hoechst}
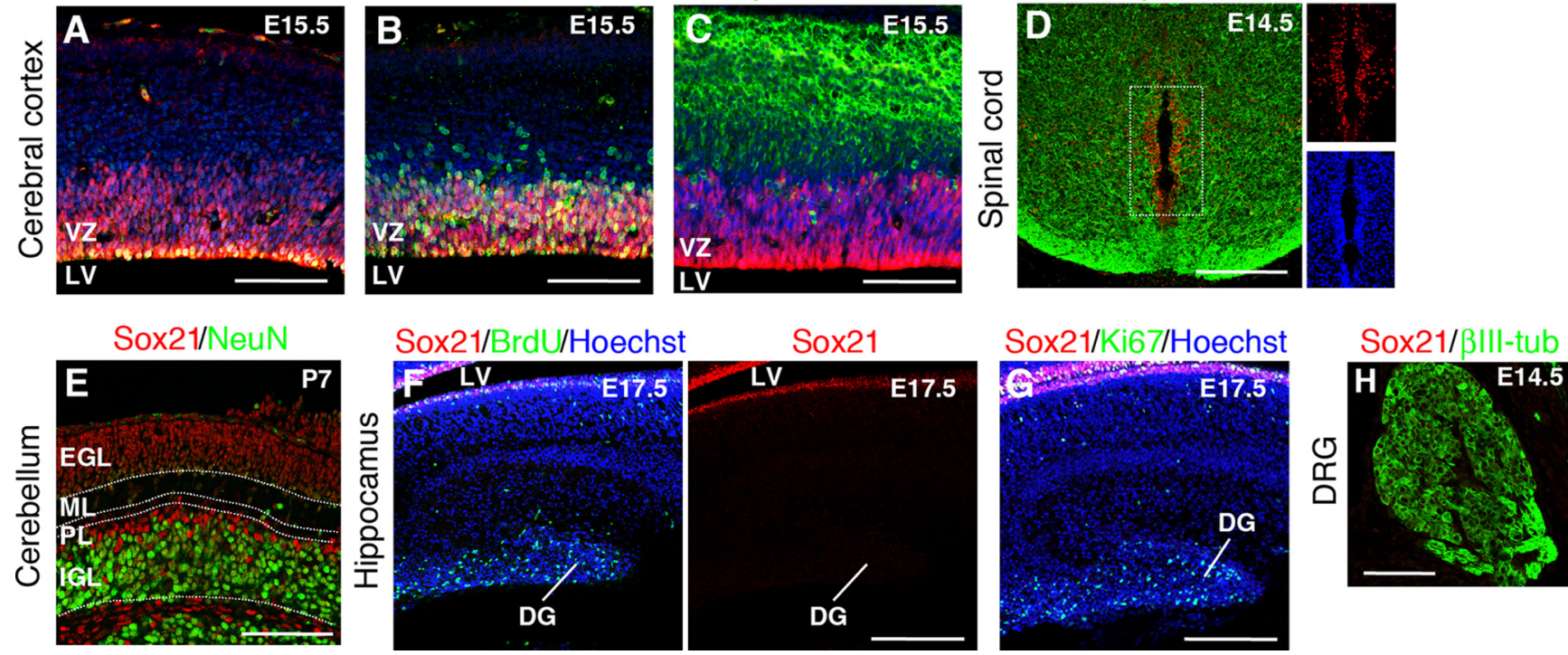

Figure 1. Expression of Sox21 in NS/PCs in the developing CNS. Immunohistochemical analyses of Sox21 in the mouse embryonic and postnatal nervous system. $A-C$, E15.5 cerebral cortex. Sox21 was expressed in Ki67-positive $(\boldsymbol{A})$ and BrdU-positive $(\boldsymbol{B})$ proliferative NS/PCs in the VZ; expression was mutually exclusive with that of the neuronal marker $\beta \| 1 I-t u b u l i n(\boldsymbol{C})$. D, E14.5 spinal cord. Sox21 was not expressed in $\beta$ III-tubulin-positive neurons. $E$, P7 cerebellum. Sox21 was highly expressed in the EGL, and expression was mutually exclusive with that of the neuronal marker, NeuN. $\boldsymbol{F}, \mathbf{G}$, E17.5 hippocampus. BrdU- and Ki67-positive NS/PCS were observed; however, Sox21 was not expressed. $\boldsymbol{H}$, E14.5 dorsal root ganglia. Sox21 was not expressed in the DRG. Scale bars: $\boldsymbol{A}-\boldsymbol{C}, \boldsymbol{E}$, $\boldsymbol{H}, 100 \mu \mathrm{m} ; \boldsymbol{D}, \boldsymbol{F}, \mathbf{G}, 200 \mu \mathrm{m}$. VZ, Ventricular zone; LV, lateral ventricle; EGL, external granular layer, ML, molecular layer; PL, Purkinje cell layer; IGL, internal granular layer; DRG, dorsal root ganglia; $D G$, dentate gyrus.

from the ChIP eluates by SDS-PAGE to obtain $\sim 100-300$ bp fragments. Size-fractionated DNA was extracted, and a single adenosine was added using Exo-Minus Klenow DNA polymerase ( $3^{\prime}$ to $5^{\prime}$ exo-minus; Illumina). Illumina adaptors were then added, and the DNA was subjected to 20 cycles of PCR according to the manufacturer's instructions. The DNA was purified, and cluster generation and 36 cycles of sequencing were performed using the Illumina cluster station and $1 \mathrm{G}$ analyzer according to the manufacturer's instructions.

The sequence reads were obtained and mapped to the mouse [mm9; from the University of California, Santa Cruz (UCSC) database; http:// genome.ucsc.edu] genomes using the Illumina Genome Analyzer Pipeline. As a result, 10,487,673 and 35,128,215 sequence reads were mapped uniquely for Sox 21 and Sox2, respectively. For each 300 bp sliding window, the mapped sequence counts for ChIP were used for the calculation. The $p$ value was also calculated, as the mapped sequences should follow a binominal distribution. The windows that passed the threshold and were located within 250 bp intervals were merged together and used for one binding site. For each binding site, the midpoint of the most significant (equal to the maximum $-\log _{10} p$ ) window was used as the peak position. To eliminate noise, regions containing $>50 \%$ repetitive sequences (identified using RepeatMasker and simpleRepeats from the UCSC database) were discarded. Calculated $p$ values were displayed using the Affymetrix Integrated Genome Browser.

Binding sites were compared using RefSeq and Known Genes from the UCSC database. Genes with binding sites within a region from $5 \mathrm{~kb}$ upstream to $1 \mathrm{~kb}$ downstream of their transcription start sites (TSSs) were annotated as bound genes. These analyses were performed using an in-house program written in Java.

De novo computational analysis of consensus sequences. MEME (Bailey and Elkan 1994) was used to search for enriched motifs among the binding sequences for both Sox 21 and Sox 2 . Nonrepetitive sequences (repetitive rate, $<10 \%$; RepeatMasker and SimpleRepeats) of $200 \mathrm{bp}$ around the position of the highest binding $p$ values were used. The MEME program was applied to 495 sequences from the Sox 21 ChIP data and 430 sequences from the Sox 2 ChIP data. The motif generated for the matched sequences $\left(p<10^{-5}\right)$ was visualized using the WebLogo program (http://weblogo.berkeley.edu). The matrix score $(0.0-1.0)$ was the highest score obtained using the position weight matrix method for each sequence (Kel et al., 2003).
Luciferase reporter analysis. To generate the reporter constructs, Venusfirefly luciferase (Luc) and/or a $\beta$-globin minimal promoter (pBG) fragment were introduced into the pGL3-basic vector (Promega). The Hes5 genomic upstream regions ( -2767 to $+73 \mathrm{bp}$ [distal regulatory element (DRE) and proximal promoter], -688 to $+73 \mathrm{bp}$ [proximal promoter], or -2767 to $-2244 \mathrm{bp}$ [DRE] from the TSS) of mouse genomic DNA were inserted into pGL3-Luc or pGL3-pBG-Luc vector. A point mutation in the Sox-binding sequence was introduced using a Mutagenesis kit (Sigma-Aldrich) and the following primers: 5' -CAGTGTTAAACGGCCCAAGCTTGCCAGGCGTG TGCCT-3' and 5'-GGCCGTTTAACACTGTGAATGGGGCCAAAG-3' (underlined letters indicate the mutated bases). The nucleotide sequences of these constructs were confirmed by DNA sequencing. Luc reporter constructs were cotransfected into AHP cells ( $80 \%$ confluent in 24 -well plates) using expression vectors encoding Sox21, Sox2, and/or Notch intracellular domain (NICD; CMV-driven) and the FuGENE 6 transfection reagent (Roche). The amount of the reporter construct relative to the internal control vector was 12:1. Luc activity was assayed $24 \mathrm{~h}$ after transfection using a dual-luciferase reporter assay system (Picka-Gene Dual; Toyo Ink). A Renilla Luc vector driven by a TK promoter was used to normalize the transfection efficiency.

$R N A$ extraction and RT-PCR. To isolate retrovirus-overexpressing AHP cells, cells were harvested $1.5 \mathrm{~d}$ after retroviral infection using $0.25 \%$ trypsinEDTA, suspended in HBSS containing $10 \mathrm{mg} / \mathrm{ml}$ propidium iodide (PI), and filtered through a $30 \mu \mathrm{m}$ mesh. The cells were sorted using a FACSVantage SE (Becton Dickinson) or a MoFlo Cell Sorter (Beckman Coulter), and viable infected cells (PI negative and GFP positive) were collected. To detect endogenous mRNA expression levels, hippocampal tissues from 6-week-old mice were dissected in cold PBS under a microscope using a scalpel. The cells or tissues were collected, and total RNA was isolated from GFP-sorted AHP cells or the hippocampus using TRIzol reagent (Invitrogen) and the Qiagen RNeasy Micro kit. cDNA was synthesized using Superscript III reverse transcriptase (Invitrogen). qPCR was performed in duplicate using SYBR green on a Stratagene MX3000P thermal cycler (Stratagene). The amount of cDNA was determined from the threshold cycle value after normalization with gapdh mRNA. Electrophoresis of all PCR products resulted in a single band of the correct size. Primer sequences were as follows: rat Hes5, 5'-ATGCTC AGTCCCAAGGAGAAAAAT- 3 ' and 5'-CAGGACTACAGCGAGGGTTA CTC-3' ' mouse Hes5, 5' -AGAAAAACCGAGTAAGTGCAACTC- ${ }^{\prime}$ ' and 5' 

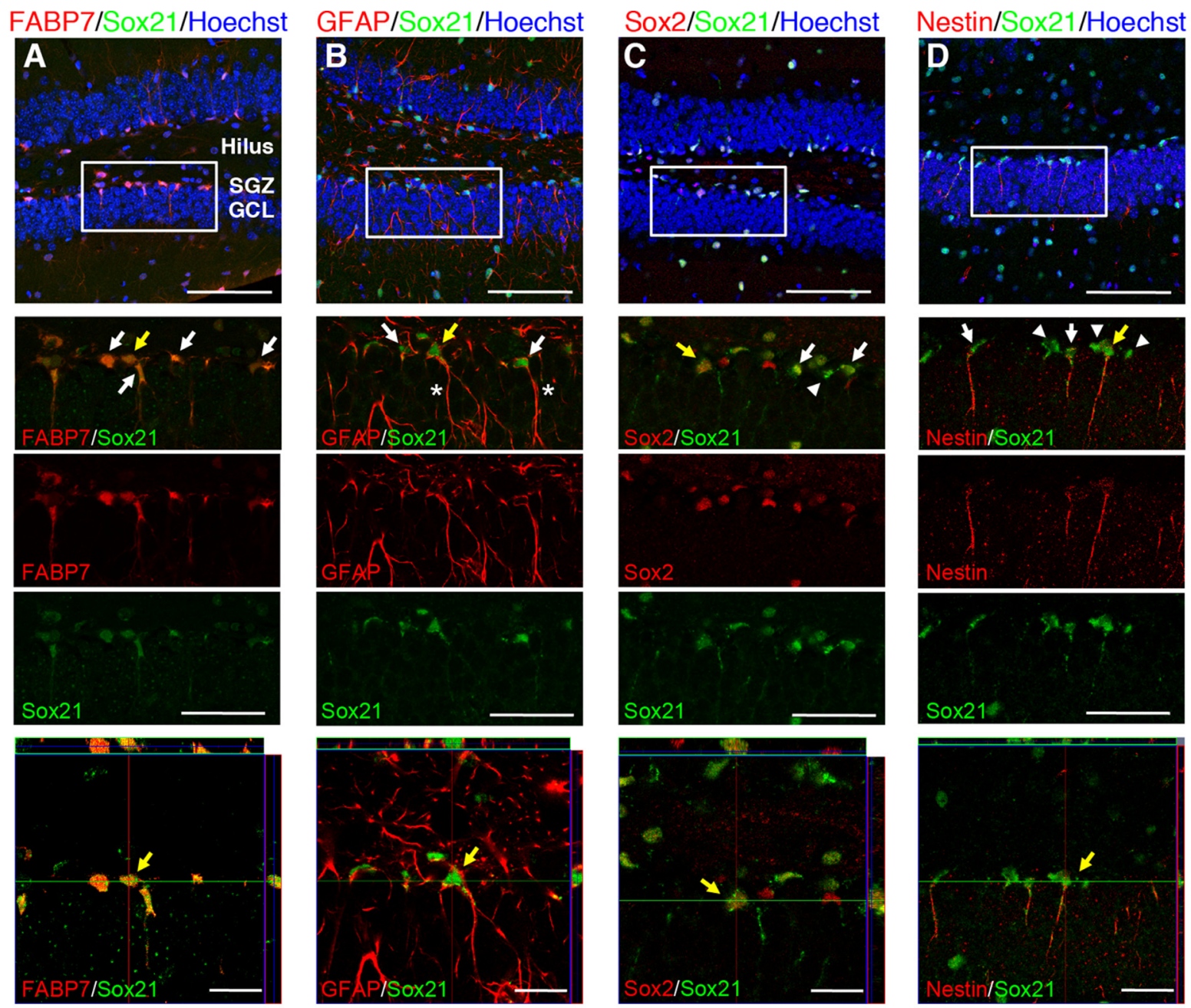

PSA-NCAM/Sox21/Hoechst

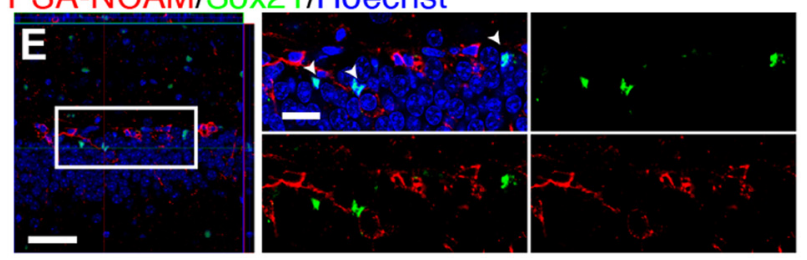

NeuN/Sox21/Hoechst

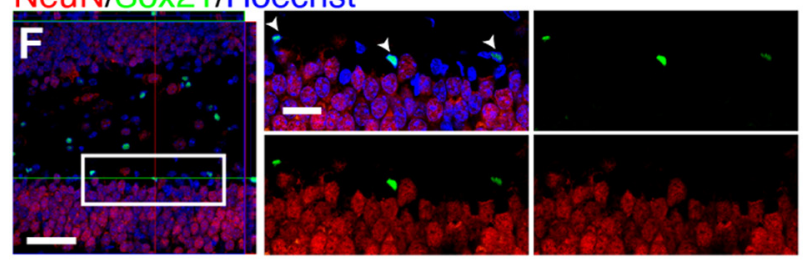

Sox21/S100ß/Hoechst Sox21/GST $\pi /$ Hoechst
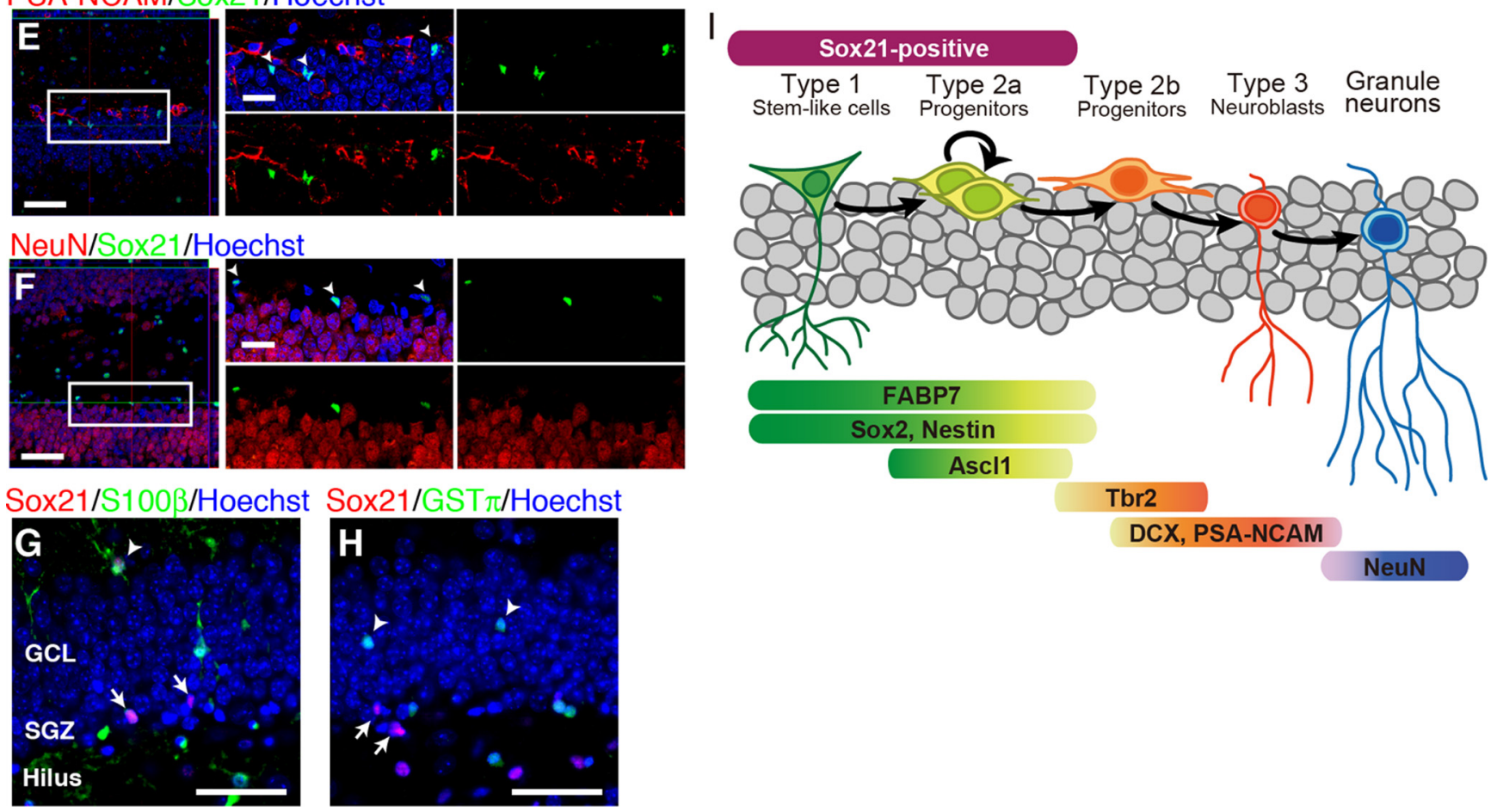

NeuN

Figure 2. Specific expression of Sox21 in NS/PCs in the adult DG. Immunohistochemical analyses of Sox 21 in the adult DG. $A-D$, The areas surrounded by white rectangles in the top panels are shown in the magnified views in the middle three panels. Arrows indicate Sox21 and marker double-positive cells; arrowheads indicate Sox21 single-positive cells. The (Figure legend continues.) 
CACCTTTGCTGTGTTTCAGGTAG-3'; and mouse/rat Gapdh, 5' -ACCA CAGTCCATGCCATCAC- $3^{\prime}$ and 5' $^{\prime}$-TCCACCACCCTGTTGCTGTA- ${ }^{\prime}$.

Knockdown of Hes5. The following four oligonucleotides were designed using the siDirect design program and used to knockdown mouse Hes5 as Hes5-specific short hairpin RNAs: $5^{\prime}$-GCAGATGAAGCTGCTT TAA-3', 5' -GATGCTCAGTCCCAAGGAA-3', 5' -TCCTCCGCTAAGG CTGCTA-3' ${ }^{\prime}$, and $5^{\prime}$-CCAGCGACACGCAGATGAA- $3^{\prime}$. The pSilencer 2.1-U6 negative control (Ambion) sequence was used as a negative control. The efficiency and specificity of these sequences were evaluated by Western blotting of stable NIH3T3 transformants expressing mouse Hes 5 normalized to $\alpha$-tubulin expression.

Western blotting. Western blotting was performed using standard protocols (Tada et al., 2007) with anti-Flag M2 (mouse IgG; Sigma-Aldrich, F3165; $1: 1000$ ) and anti- $\alpha$-tubulin (mouse IgG; Sigma-Aldrich, T9026; 1:5000) antibodies. The blots were developed using ECL reagents (GE Healthcare) and the SuperSignal West Dura Extended Duration Substrate (Pierce).

Microscopy and quantification. To quantify the cell type of Sox21positive cells in the adult DG, at least 20 Sox $21+$ cells in each mouse were detected, and the percentage of marker $+/$ Sox $21+$ cells among the total Sox $21+$ cell population was calculated. Values were expressed as the mean $\pm \mathrm{SE}(n=3)$. For the quantitative analyses of marker coexpression, $1.5 \mu \mathrm{m}$ sliced $z$-stacking images of at least five vibratome-cut coronal sections (50 $\mu \mathrm{m}$ thick) were analyzed in every sixth section using LSM510 and LSM700 confocal microscopes (Zeiss), and the numbers of cells double positive for stage-specific markers (FABP7, GFAP, Ascl1, Tbr2, DCX, or NeuN) and BrdU were counted. To quantify Sox2- and Ki67-positive cells in the P7 hippocampus, the number of immunopositive cells per $0.03 \mathrm{~mm}^{2} \mathrm{DG}$ was counted and expressed as cell number per $\mathrm{mm}^{2} \mathrm{DG}$.

\section{Results}

\section{Sox 21 is expressed in NS/PCs in developing and adult nervous systems}

We demonstrated previously the restricted expression of SoxB1 and Sox 21 proteins in neurogenic regions in both embryonic and adult brains (Tada et al., 2007). To reveal the detailed expression pattern of Sox 21 in the nervous system, we performed immunohistochemical analyses with antibodies directed against cell type markers and by BrdU labeling. Sox 21 was exclusively expressed in the ventricular zone of the E15.5 cerebral cortex, which was populated by BrdU-incorporated and Ki67-positive NS/PCs but not $\beta$ III-tubulin-positive neurons (Fig. $1 A-C$ ). Similar Sox21 expression patterns were also observed in the NS/PC population in the E14.5 spinal cord and P7 cerebellum (Fig. 1D,E). In E17.5 hippocampus, however, Sox21 expression was not detected, even in BrdU-incorporated or Ki67-positive cells (Fig. $1 F, G$ ), which contrasted with the expression pattern of NS/PCs in the ventric-

\section{$\leftarrow$}

(Figure legend continued.) cells highlighted by yellow arrows are also shown using orthogonal views at higher magnification in the bottom panels (top planes are through the $x-z$ axes, and right planes are through the $y$ - $Z$ axes). In the DG, Sox21 expression was restricted to cells in the SGZ. Sox21 was expressed in FABP7-positive ( $\boldsymbol{A}$ ) and GFAP-positive (B) NS/PCs with horizontally oriented cell bodies typical of type 2a cells and also in GFAP-positive stem-like cells with radial glia-like fibers ( $\boldsymbol{B}$, asterisk) typical of type 1 cells. $\boldsymbol{C}, \boldsymbol{D}$, The Sox21 expression pattern showed incomplete concordance with the other NS/PC markers, Sox2 ( $C$ ) and Nestin (D). $\boldsymbol{E}$, Very few Sox21-positive cells coexpressed PSA-NCAM, a marker for immature neurons. Arrowheads indicate Sox21 single-positive cells. $\boldsymbol{F}$, The Sox21-positive cell population was mutually exclusive of NeuN-positive neurons in the DG. Arrowheads show Sox21 single-positive cells. $\mathbf{G}, \boldsymbol{H}$, Sox21 was not expressed in S100 $\beta$-positive astrocytes or GST $\pi$-positive oligodendrocytes in the DG, although some $S 100 \beta$-positive cells outside the GCL (arrowhead) expressed Sox21. I, A schematic summary of neuronal differentiation in the adult $D G$ and the markers expressed at each stage. Scale bars: $\boldsymbol{A}-\boldsymbol{D}$, top, $100 \mu \mathrm{m}$; middle, $50 \mu \mathrm{m}$; bottom, $20 \mu \mathrm{m} ; \boldsymbol{E}, \boldsymbol{F}$ (panels with orthogonal views) $\boldsymbol{G}, \boldsymbol{H}, 50 \mu \mathrm{m} ; \boldsymbol{E}, \boldsymbol{F}$ (magnified views), $20 \mu \mathrm{m}$. ular zone. Sox21 was not observed in E14.5 dorsal root ganglia (Fig. $1 H$ ).

We next examined the expression of Sox 21 in the adult nervous system, particularly focusing on the hippocampus, which continuously generates new neurons. As we reported previously, Sox21 was expressed in the SGZ of the adult hippocampal DG (Tada et al., 2007) (Fig. 2). To further analyze the differentiation stage of Sox21-positive cells in the DG, immunohistochemical analyses using specific antibodies against Sox 21 and cell-typespecific markers were performed. A large portion of Sox21positive cells expressed FABP7 (73.2 $\pm 1.9 \%$; Fig. $2 A)$ and GFAP (81.6 $\pm 3.4 \%$; Fig. $2 B)$, both of which are markers for type 1 and 2 a cells. Some Sox 21-positive cells possessed radial glia-like fibers traversing the entire GCL (Fig. $2 B$, asterisks), a morphology typical of type 1 cells (Seri et al., 2001). Sox21 was also expressed in FABP7- or GFAP-positive cells in the hilus (Fig. 2A,B). Compared with FABP7 and GFAP, Sox21 expression showed less overlap with the other markers for type 1 and 2a cells, Sox2 (58.5 $\pm 9.4 \%$; Fig. $2 C)$, and Nestin (39.9 $\pm 4.5 \%$; Fig. $2 D)$. Sox 21 was rarely coexpressed with PSA-NCAM, a marker for immature neurons $(1.59 \pm 1.59 \%$; Fig. $2 E)$, but was exclusively expressed with NeuN, a marker for mature neurons (Fig. $2 F$ ). In addition, Sox 21 was not expressed in astrocytes (S100 $\beta$ positive) or oligodendrocytes (glutathione $S$-transferase $\pi$ positive) in the adult DG or hilus (Fig. 2G,H). Together, these results indicated that Sox 21 was expressed in a portion of type 1 stem-like cells and type 2a transient amplifying progenitors (Fig. $2 I$ ).

\section{Adult neurogenesis, but not embryonic neurogenesis, is impaired in Sox $21^{-1-}$ mice}

Because Sox 21 was highly expressed in NS/PCs in both the embryonic and adult nervous systems (Fig. 1,2), we next examined whether Sox 21 plays a role in neurogenesis. To examine the function of Sox21 in vivo, Sox21-deficient/GFP knock-in (Sox21 $\left.1^{-/}\right)$ mice (Kiso et al., 2009) were generated. In the P3 cerebral cortex of Sox $21^{-/-}$mice, no obvious abnormality was detected in Brn2expressing layer II-IV neurons and Tbr1-expressing layer VI neurons (Fig. $3 A, B$ ). Although Sox 21 was highly expressed in the external GCL, which comprises precursor cells of granule neurons in P7 cerebellum (Fig. 1E), the thickness and cell density of the granule cell layer appeared normal in adult Sox $21^{-1-}$ cerebellum (Fig. 3C). Furthermore, consistent with the undetectable level of Sox 21 expression in the embryonic hippocampus, the gross structure of the hippocampus was normal in P7 Sox $21^{-1-}$ mice (Fig. 3D). The numbers of Ki67- or Sox2-positive NS/PCs and NeuN-positive neurons that differentiated between the embryonic and neonatal stages were also not impaired in $\mathrm{P} 7$ Sox $21^{-1-}$ hippocampus (Fig. $3 E-G$ ). These results indicate that Sox 21 may not play a crucial role in developmental neurogenesis in mice, at least in the regions that we analyzed.

We next examined the role of Sox 21 in adult hippocampal neurogenesis using a well-established BrdU labeling strategy for the detailed analysis of adult neurogenesis in the subventricular zone and DG (Sakaguchi et al., 2006; Imaizumi et al., 2011). The long-term administration of BrdU followed by a 4 week washout period allows the retrospective analysis of the differentiation stage of BrdU-retaining cells (Fig. 4A). We first quantified the number of type 1 cells that retained BrdU, expressed FABP7 or GFAP, and also possessed radial glia-like fibers (which is a feature of type 1 cell morphology) in the SGZ in wild-type and Sox $21^{-/-}$ mice. As shown in Figure 4, $B$ and $C$, the number of type 1 cells was not significantly changed in Sox $21^{-1-}$ mice. The number of proliferating type 1 cells that expressed Ki67 was also unchanged 

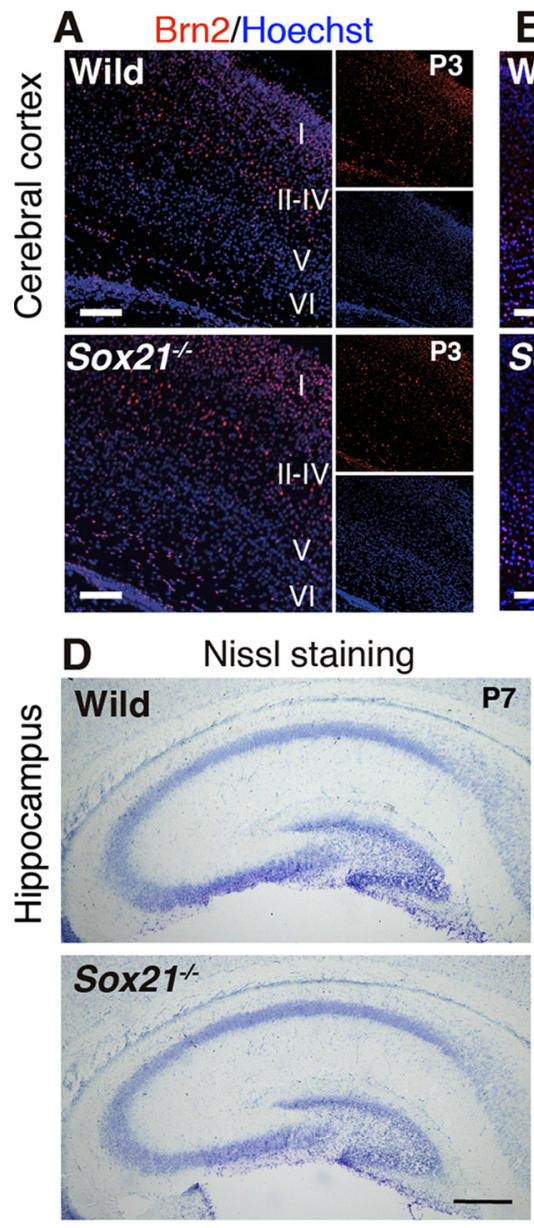

B Tbr1/Hoechst
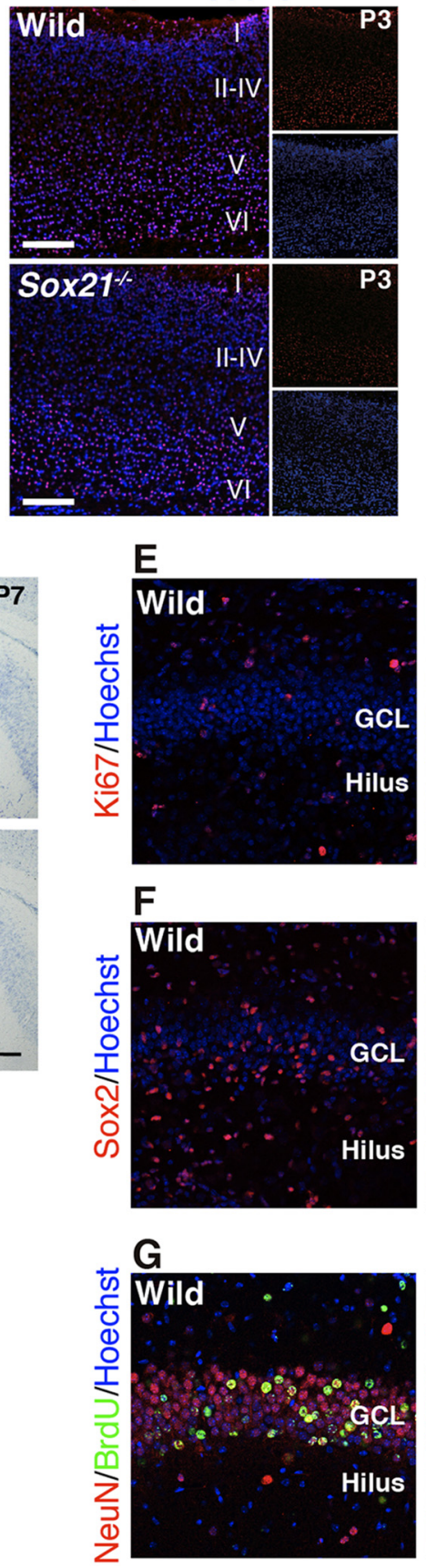
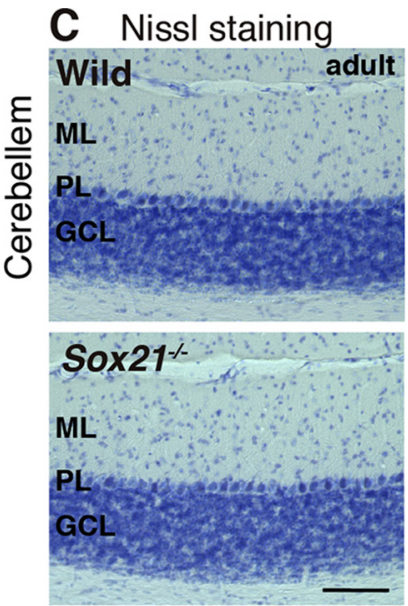
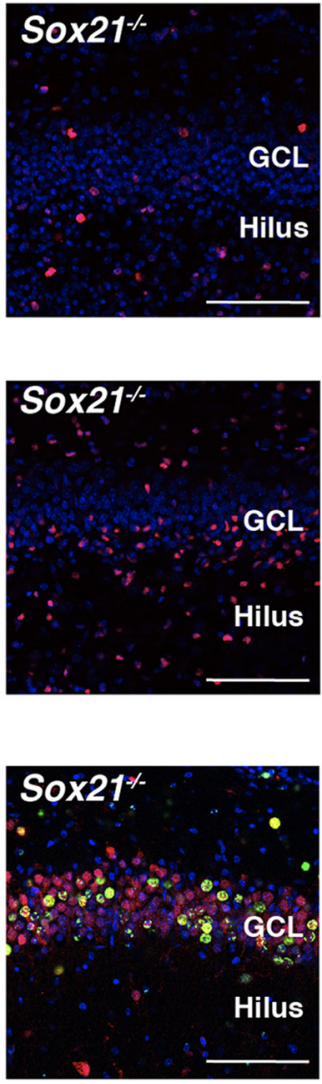
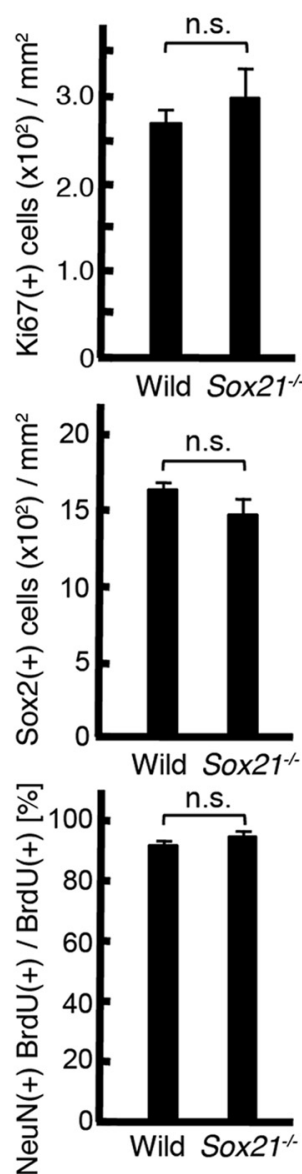

Figure 3. Normal neuronal development in Sox $21^{-/-}$mice. $A, B$, Distribution of layer-specific neuronal markers in the cortex of wild-type and Sox $21^{-/-}$mice at P3. Brn2 (layer II-IV; $A$ ) and Tbr1 (layer Vl; $\boldsymbol{B}$ ) expression patterns in Sox2 $1^{-1-}$ mice were similar to those in wild-type mice. $\boldsymbol{C}$, In the adult cerebellum, no impairment was observed in GCL thickness or the cell density in PL and ML. D, The hippocampal structure was normal in P7 Sox2 $1^{-/-}$mice. $\boldsymbol{E}, \boldsymbol{F}$, The numbers of Ki67-positive $(\boldsymbol{F})$ and Sox2-positive $(\boldsymbol{F})$ NS/PCs in the hippocampus were not altered in P7 Sox2 ${ }^{-/-}$mice. G, BrdU was administered at E15.5. BrdU-retaining NeuN-positive cells in P7 Sox $21^{-/-}$mice were comparable with those in wild-type mice. Data represent the mean \pm SE. n.s., No significance. Scale bars: $A-C, E-G, 100 \mu \mathrm{m} ; \boldsymbol{D}, 300 \mu \mathrm{m}$. Abbreviations are as in Figure 1.

in Sox $21^{-1-}$ mice $[0.38 \pm 0.04$ cells (wild type) vs $0.35 \pm 0.06$ cells $\left(\right.$ Sox $\left.21^{-1-}\right)$ per DG field; not significant; $n=5$ ]. These results suggest that the deficiency of Sox 21 did not influence the type 1 cell compartment.

To examine their efficiency for differentiating into neurons, we next compared the number of BrdU-retaining cells that expressed the immature neuronal marker DCX and the mature neuronal marker NeuN in wild-type and Sox $21^{-/-}$mice. After the 4 week BrdU washout, the number of BrdU and NeuN double-positive cells was significantly reduced in Sox $21^{-/-}$mice (Fig. 4D), while that of BrdU and DCX double-positive cells did not change (Fig. $4 E$ ), suggesting that deletion of Sox21 impaired the generation of new neurons.

As the long-term BrdU treatment experiments showed that Sox 21 deficiency resulted in fewer newly generated neurons without a defect in the type 1 stem cell population, the course of neuronal differentiation in Sox $21^{-/-}$mice was likely impeded. Therefore, we next examined which differentiation stage was spe- 

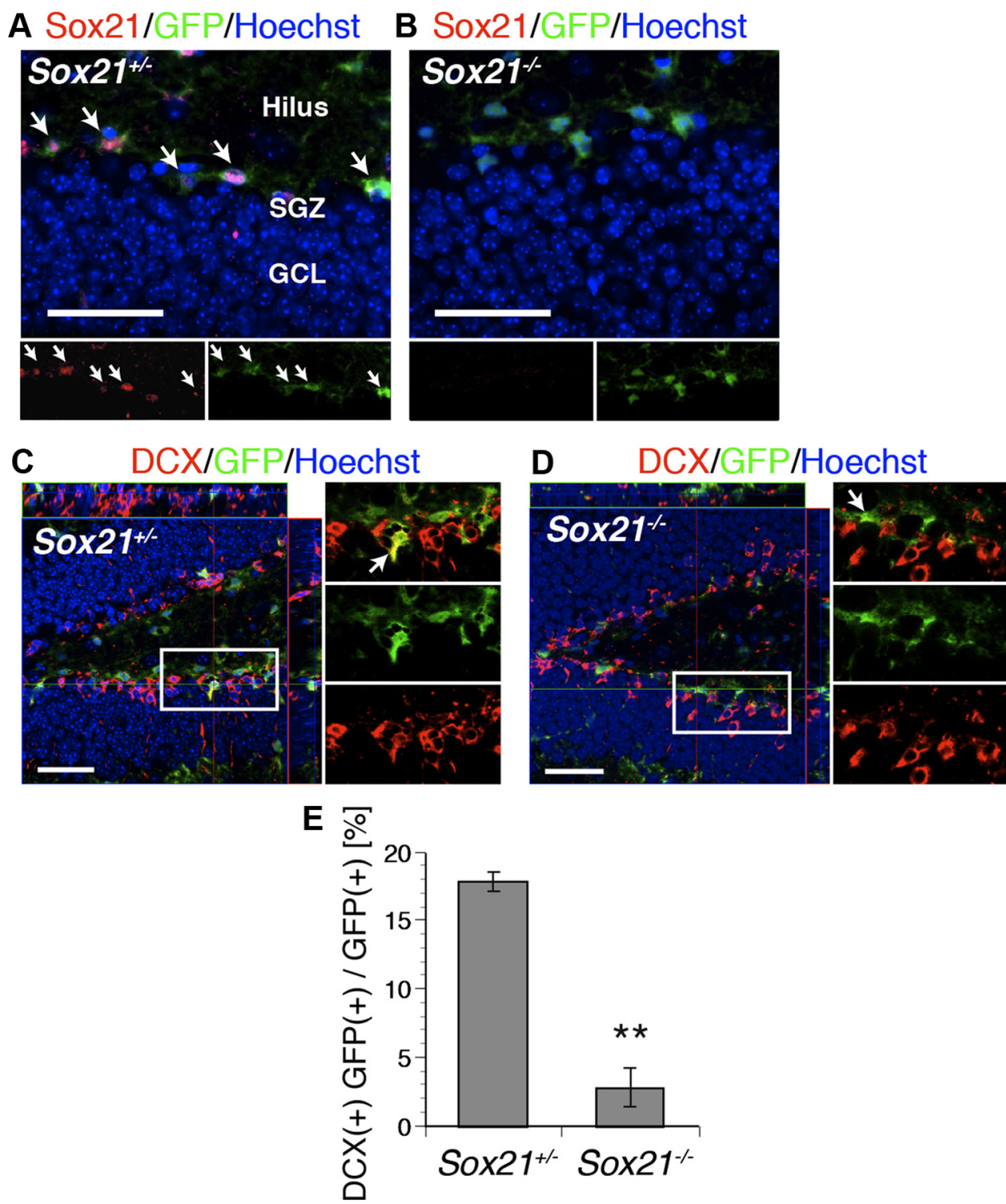

Figure 5. Lineage tracing analysis of Sox21-expressing and Sox21-deficient cells in the adult DG. $A, B$, GFP expression detected in the SGZ. $A$, GFP was detected in all Sox21-positive cells (arrows) in Sox $21^{+/-}$mice. $\boldsymbol{B}$, Sox21 expression was abolished in Sox $21^{-/-}$mice. $C, D$, Double staining for GFP and the immature neuronal marker DCX. Top planes are through the $x-z$ axes, and right planes are through the $y$-z axes. Magnified views are shown in the right panels. Some GFP + cells ( $\boldsymbol{C}$, arrows) colocalized with DCX + cells in Sox $21^{+/-}$mice; however, GFP and DCX expression showed less overlap in Sox $21^{-/-}$mice $\left(\boldsymbol{D}\right.$, arrows). $\boldsymbol{E}$, The proportion of DCX and GFP double-positive cells within the total GFP-positive cell population in the DG of Sox21 ${ }^{+/-}$and Sox $21^{-/-}$mice. Scale bars: $50 \mu \mathrm{m}$. Data represent the mean $\pm \mathrm{SE}$. ${ }^{* *} p<0.01$ (two-sided $t$ test). $n=3$.

ities were detected in the neuron and NS/PC compartments in the neonatal Sox $21^{-1-}$ hippocampus (Fig. 3), the defect in adult neurogenesis in the Sox $21^{-/-}$hippocampus is, conceivably, much less likely to be affected by developmental defects.

\section{Sox 21 overexpression promotes neuronal differentiation}

To determine whether Sox 21 is capable of promoting neuronal differentiation, a gain-of-function assay was performed in vivo. A retrovirus was stereotactically introduced into the DG of adult wild-type mice to induce expression of GFP and/or Sox 21 in NS/PCs (Fig. 6A). Infection-derived GFP expression was restricted to the SGZ at $3 \mathrm{~d}$ after retrovirus infusion, which enabled monitoring of the fate of the NS/PCs (Fig. 6B). At $28 \mathrm{~d}$ after infusion of the retrovirus suspension, the majority of GFPpositive infected cells were found to reside in the GCL and SGZ (Fig. $6 C, D$ ). Among the control virus-infected cells, $11.5 \pm 1.5 \%$ of the GFP-positive cells expressed the neuronal marker NeuN, while a significantly larger proportion $(60.0 \pm 5.9 \%)$ of GFPpositive cells in the Sox 21 -overexpressing group expressed NeuN (Fig. 6F). These results strongly support the hypothesis that Sox 21 is an important positive regulator of the neuronal differentiation of NS/PCs in the adult DG.

\section{The Hes5 gene is a direct target of Sox 21}

To elucidate the molecular mechanism underlying Sox $21-$ mediated regulation of neurogenesis, ChIP sequencing was performed using embryonic NS/PC cultures to identify the target genes of Sox 21 and Sox2. Neurosphere cultures from the forebrains of embryonic mouse embryos at E11.5 were used as the cell source, because a large homogenous cell population was required to perform ChIP sequencing. After sequencing all genomic DNA that bound to endogenous Sox 21 or Sox 2 proteins, 284 regions from the Sox 21-ChIP sequencing data and 301 regions from the Sox2-ChIP sequencing data were identified by comparison with 
A retrovirus construction

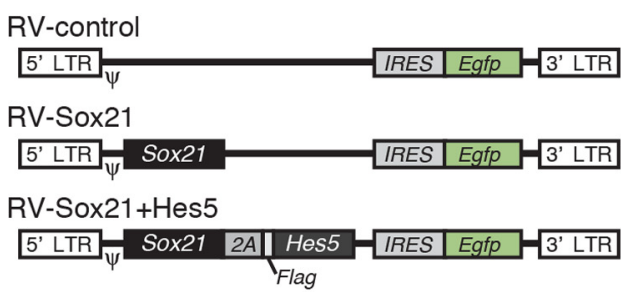

C NeuN/GFP/Hoechst
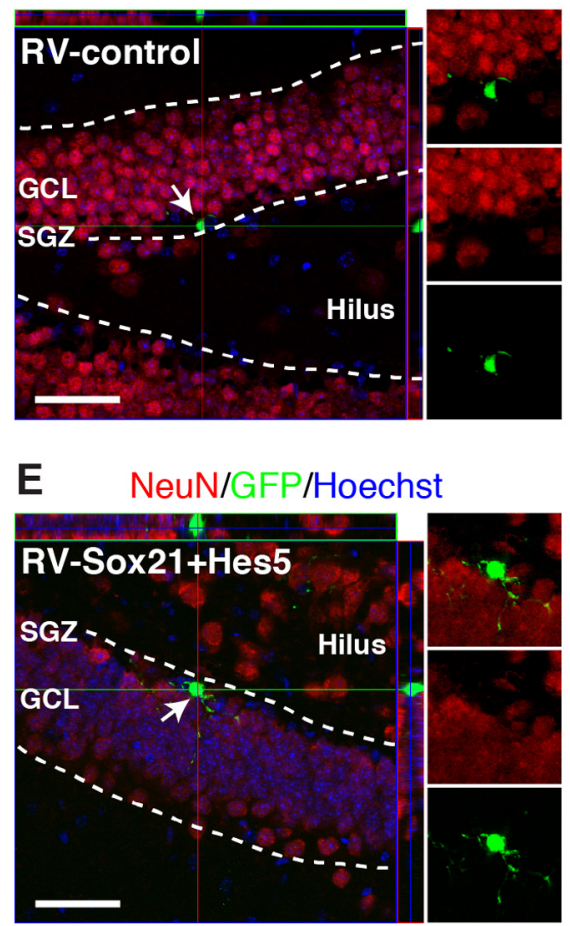

B

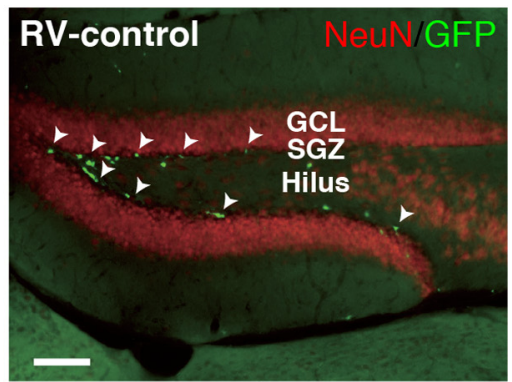

D NeuN/GFP/Hoechst

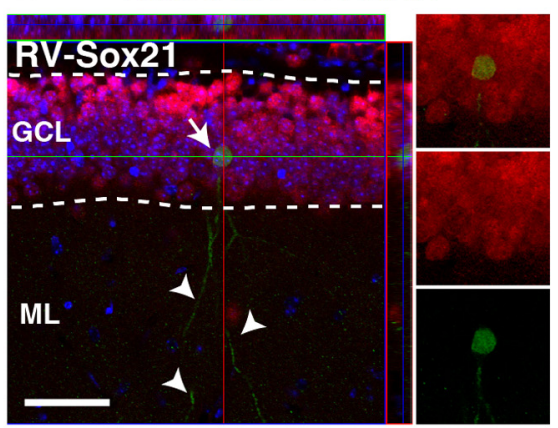

$\mathbf{F}$

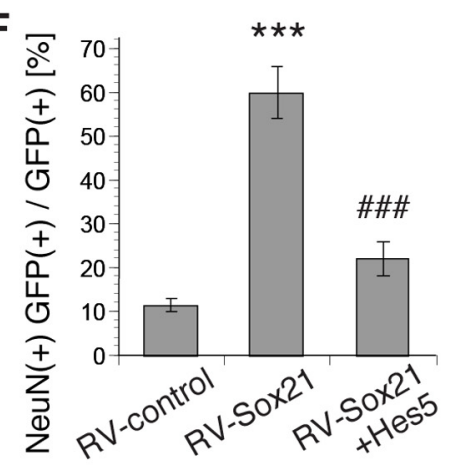

Figure 6. Sox21 overexpression promoted neuronal differentiation of newborn cells in vivo. A, Schematic showing control-, Sox21-, and Sox21-2A-Hes5-overexpressing retroviral (RV) constructs. $\psi$ depicts a packaging signal used to generate the retrovirus. $2 A$ depicts self-cleaving $2 A$ peptides originating from the foot-and-mouth disease virus. B, Micrographs of GFP-positive cells $3 \mathrm{~d}$ after retrovirus infusion into the $D G$ of 6-week-old wild-type mice. The retrovirus infused into the $D G$ delivered its transducing GFP gene selectively into NS/PCS in the SGZ of the hippocampus (arrowheads). $C-E$, Micrographs showing retrovirus-labeled GFP-positive newborn cells (arrows) $28 \mathrm{~d}$ after retrovirus infusion into the DG of 6-week-old wild-type mice in the the controls $(\boldsymbol{C})$, the Sox21-overexpressing group (D), and the Sox21-2A-Hes5-overexpressing group ( $\boldsymbol{E}$ ). The GFP-positive infected cell in $\boldsymbol{D}$ represents a mature granule neuron showing NeuN expression and long branches (arrowheads) in the ML of the hippocampus, whereas the majority of GFP-positive cells in the SGZ in $\boldsymbol{C}$ and $\boldsymbol{E}$ lacked NeuN expression and exhibited horizontally expanding branches typical of NS/PCS. F, Quantification of neuronal differentiation in GFP-positive cells. The data represent the proportion of NeuNpositive cells within the total GFP-positive infected cell populations in the SGZ and GCL. Data represent the mean \pm SE. ${ }^{* * *} p<$ 0.001 ; $\# \#$ \# 0.001 (two-sided $t$ test). $n=6-8$ ). Scale bars: $B, 100 \mu \mathrm{m} ; C-E, 50 \mu \mathrm{m}$.

the input DNA sequencing data. Among them, 97 genomic loci were bound by both Sox 21 and Sox2, which accounted for 34.5 and $32.2 \%$ of the regions bound by Sox 21 and Sox 2 , respectively (Fig. 7A). The genes in which TSSs resided near the $p$ value peak were then selected as the candidate targets (40 example target genes are listed in Table 1).

Among the candidate target genes of Sox 21 and Sox 2 , the Hes 5 gene, encoding Hairy and enhancer of split 5 (Akazawa et al., 1992), which acts as a downstream effector of Notch signaling, was selected because it contains Sox-consensus sequences (Fig. $7 B$ ) and maintains the undifferentiated state of NS/PCs and represses their neuronal differentiation (Ohtsuka et al., 1999). Sox 21 and Sox 2 bound to the Hes 5 gene at -312 to +10 bp from the TSS (Fig. 7C,D, bottom sequence). This area is highly conserved among various species and exhibits promoter activity for Hes5 gene expression (Takebayashi et al., 1995). The maximum $p$ values $\left(-\log _{10} p\right)$ for Sox 21 and Sox 2 were 79.8 and 33.0, respectively. Surprisingly, another Sox21 binding site (maximum $\left.-\log _{10} p=73.4\right)$ was identified at -2576 to -2335 bp from the TSS (Fig. $7 D$, top sequence). This region is also evolutionarily conserved but demonstrated no notable signal in the Sox2-ChIP sequencing data. Because the two regions covered by Sox 21 contained the CBF/Suppressor of Hairless/Lag1 (CSL)- and Sox-consensus sequences (Fig. 7D), both regions may be involved in transcriptional regulation. In subsequent experiments, these regions were defined as a well-known proximal promoter and a novel DRE.

Because the ChIP sequencing data shown here were obtained from cultured embryonic NS/PCs, ChIP-quantitative PCR analyses were also performed using an AHP cell line (Palmer et al., 1997) to validate the occupation of the Hes5 gene upstream regions by Sox 21 and/or Sox 2 in "adult"-type NS/PCs. The results were very similar to those obtained from ChIP sequencing using embryonic NS/PCs. Sox 21 bound to both the proximal promoter and DRE, whereas Sox 2 only bound to the proximal promoter in AHP cells (Fig. 7E). Thus, the results of the ChIP sequencing and ChIP-quantitative PCR analyses suggest that the Hes 5 gene may be a potential target of Sox 21 in adult NS/PCs.

\section{Sox 21 represses Hes 5 expression in adult NS/PCs}

The effect of Sox 21 on Hes 5 gene promoter activity was analyzed using reporter experiments. The Hes5 upstream genomic region ( -2767 to $+73 \mathrm{bp}$ from the TSS) containing the proximal promoter and DRE was cloned into luciferase reporter plasmids and transfected with vectors carrying NICD or Sox21 into AHP cells. NICD-induced Hes 5 promoter-luciferase activity was reduced by Sox 21 coexpression in a dose-dependent manner (Fig. $8 \mathrm{~A}$, open columns). Intriguingly, introducing a mutation into the Sox21binding sequence in the DRE (Fig. $8 \mathrm{~A}$, closed columns) completely canceled the repressive effect of Sox 21 . Furthermore, the repressive effect of Sox 21 was mediated entirely by the DRE alone ( -2767 to -2244 bp from TSS; Fig. 8 C), and Sox 21 did not affect luciferase activity by binding to the Hes 5 proximal promoter $(-688$ to $+73 \mathrm{bp}$ from the TSS; Fig. $8 B$ ). These results strongly suggest that Sox 21 directly represses Hes 5 expression by binding to the DRE. We then investigated whether Sox 2 competed with Sox 21 to regulate Hes5. Although both Sox 21 and Sox2 were capable of binding to the Hes5 promoter, in sharp contrast to 


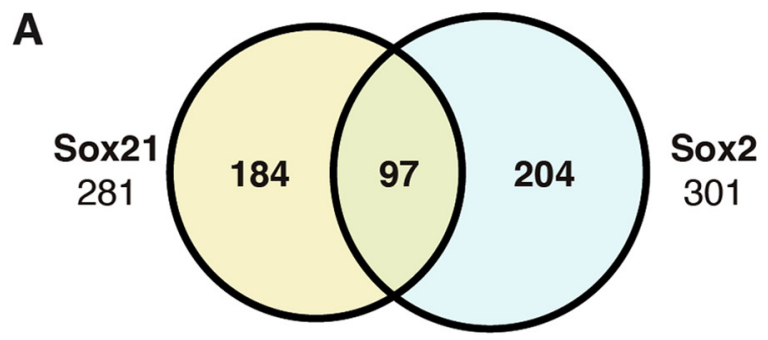

\section{Hes5 DRE sequence (mouse)}
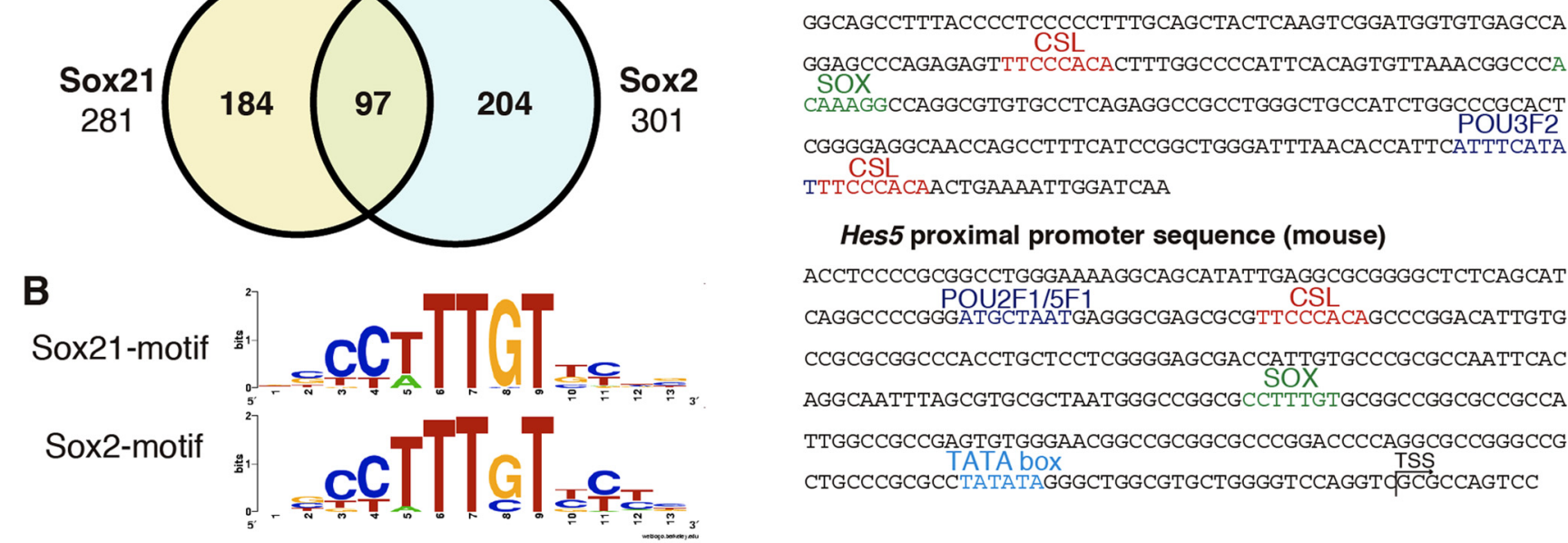

Hes5 proximal promoter sequence (mouse)

ACCTCCCCGCGGCCTGGGAAAAGGCAGCATATTGAGGCGCGGGGCTCTCAGCAT POU2F1/5F1
CAGGCCCCGGGTGCTAATGAGGGCAGCGCGTTCCCACAGCCCGGACATTGTG CCGCGCGGCCCACCTGCTCCTCGGGGAGCGACCATTGTGCCCGCGCCAATTCAC AGGCAATTTAGCGTGCGCTAATGGGCCGGCGCCTTTGTGCGGCCGGCGCCGCCA TTGGCCGCCGAGTGTGGGAACGGCCGCGGCGCCCGGACCCCAGGCGCCGGGCCG TATA box
CTGCCCGCGCCTATATAGGGCTGGCGTGCTGGGGTCCAGGT ISCGCCAGTCC
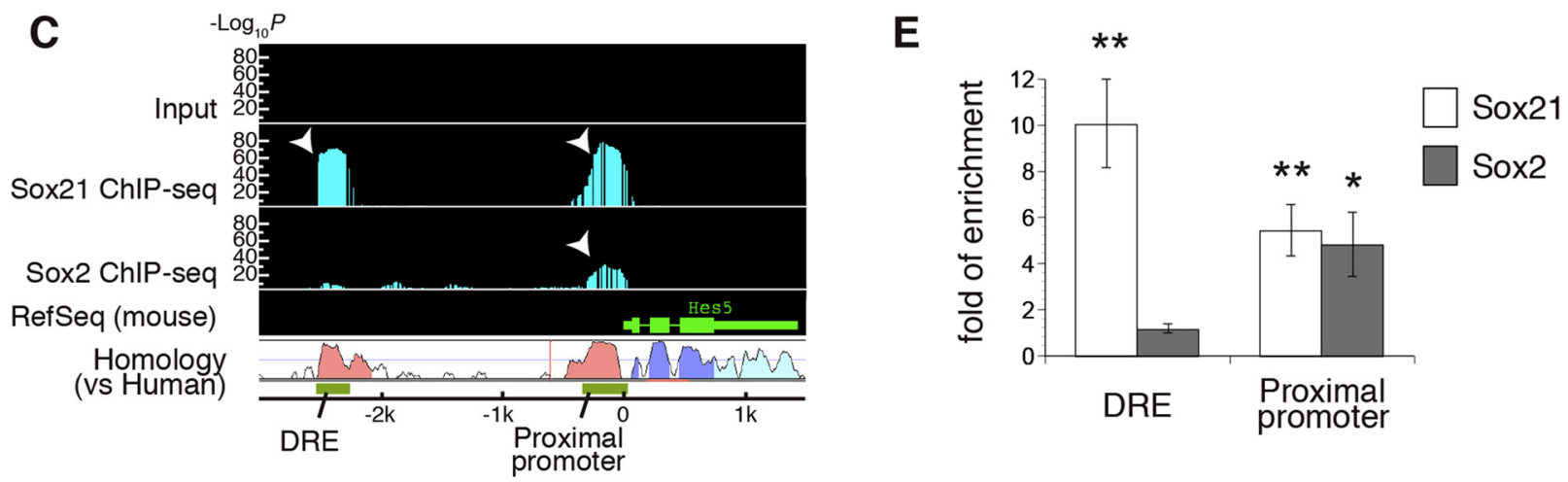

Figure 7. Identification of the Hes5 gene as a target of Sox21 using ChIP sequencing analysis. A, Venn diagram showing the number of genomic loci bound by Sox21 and Sox2. Of all the binding sites, 97 common genomic loci were bound by both Sox21 and Sox2. B, Enriched motifs in Sox21-and Sox2-binding sequences identified by de novo computational analysis of ChIP sequencing data. The height of each character represents the relative frequency of the nucleotide appearance in the binding motifs. $\boldsymbol{C}$, Genomic view of the ChIP sequencing data around the Hes 5 gene. Binding by Sox21 and Sox 2 is indicated by $P$ values $\left(-\log _{10} p\right)$ on the $y$-axis, and the regions in which $p$ values are $<10^{-10}$ are shown as green bars. Genomic sequence conservation is depicted by the graph showing homology between the mouse and human genomes. Two highly conserved regions upstream of the Hes 5 gene corresponded to the positions of the binding sites (arrowheads). $D$, Genomic sequences of the mouse Hes5 DRE and proximal promoter. Sox21-, Sox2-, CSL-, and POU-binding motifs were present in both regions. E, ChIP-quantitative PCR assay using rat AHP cells. Immunoprecipitated Hes 5 DRE or proximal promoter fragments were quantified using the cycle threshold (Ct) values, which were normalized to the Ct of the input DNA. The binding of Sox21 or Sox2 to the DNA was determined by the fold relationship of enrichment of the target fragments in the ChIP DNA over the internal Gapdh gene. Data represent the mean \pm SE. ${ }^{*} p<0.05$; ${ }^{* *} p<0.01$ (two-sided $t$ test). $n=3$ for Sox21; $n=4$ for Sox2.

Sox21, Sox2 did not modulate NICD-dependent luciferase activity (Fig. $8 D$ ) or cancel the repressive effect of Sox 21 on the Hes5 promoter, regardless of the dose of Sox2 expression vector used (Fig. $8 E$ ). These results suggest that Sox 21 determines the expression level of Hes5 in adult hippocampal NS/PCs independently of Sox2.

To further examine the effect of Sox 21 on Hes5 gene expression in adult NS/PCs, Hes5 mRNA levels were analyzed in gainand loss-of-function experiments. Endogenous Hes5 gene expression in AHP cells was significantly decreased by retrovirally introduced Sox21 (Fig. 9A). By contrast, the endogenous Hes5 expression level in the hippocampus of adult Sox $21^{-/-}$mice was significantly higher than that in wild-type mice (Fig. 9B). Together, these results suggest that Sox 21 acts as a transcriptional repressor of Hes5 in adult hippocampal NS/PCs.

\section{Sox 21 regulates adult neurogenesis by repressing Hes 5} expression

To confirm the repressive role of Sox 21 in Hes 5 expression in vivo, we analyzed the expression patterns of Sox 21 and Hes5 in the adult DG using Hes5-NLSlacZ knock-in mice (Imayoshi et al., 2010). In the DG of Hes5-NLSlacZ knock-in adult mice, we observed LacZ-positive cells that expressed GFAP (Fig. 10A), and only $23.6 \%$ of Sox 21 -expressing cells demonstrated LacZ expression. Consistent with the restricted expression of Sox 21 in type 1 and type 2 a cells (Fig. 2), Sox 21 and LacZ double-positive cells with or without type 1 cell-like fibers expressed GFAP, whereas none expressed PSA-NCAM (Fig. $10 \mathrm{~B}$ ). These results suggested that Sox 21 and Hes5 were expressed in the adult DG in an essentially exclusive manner, although Hes 5 may not be fully repressed by Sox 21 in type 1 cells and in a portion of type 2 a cells at the stage before transition to type $2 \mathrm{~b}$ cells.

To address whether Hes 5 regulates neurogenesis downstream of Sox21, we first examined the effect of reduced Hes5 expression on neuronal differentiation in the adult DG. We performed Hes5 knockdown experiments in vivo by administering a Hes5-specific shRNA-expressing retrovirus (Fig. 11A) to the adult DG. Hes5 knockdown in the DG resulted in a significant increase in the number of NeuN-positive cells compared with that in the control shRNA group (Fig. $11 B-D$ ). Like the previously reported antineurogenic function of Hes5 in embryonic NS/PCs (Ohtsuka et al., 1999), this result indicates that Hes5 is critical for the maintenance of the undifferentiated status of NS/PCs in the adult DG.

Finally, to investigate whether Hes5 is indeed a downstream effector of Sox 21 in the regulation of adult hippocampal neuro- 
Table 1. Candidate target genes of Sox21 and Sox2

\begin{tabular}{|c|c|c|c|c|c|c|c|c|c|}
\hline \multicolumn{5}{|c|}{ Sox21 ChIP sequencing } & \multicolumn{5}{|c|}{ Sox2 ChIP sequencing } \\
\hline Accession No & Gene & Peak location & $p$ & Overlap & Accession No & Gene & Peak location & $p$ & Overlap \\
\hline NM_177753 & Sox21 & $0-5 \mathrm{~kb}$ down & 110.2 & + & NM_025434 & Mrps28 & Other intron & 76.5 & + \\
\hline NM_025434 & Mrps28 & Other intron & 82.2 & + & NM_177753 & Sox21 & $0-5 \mathrm{~kb}$ down & 66.1 & + \\
\hline NM_172990 & Pank4 & 10 k-5 kb up & 79.8 & + & NM_019514 & Trim32 & Other intron & 56.7 & - \\
\hline NM_010419 & Hes5 & $0-300$ b up & 79.8 & + & NM_010308 & Gna01 & Other intron & 52.8 & + \\
\hline NM_008972 & Ptma & $0-300$ b up & 72.6 & - & NM_178642 & Tmem16a & Other intron & 50.8 & + \\
\hline NM_175274 & Ttyh3 & $10 \mathrm{k}-5 \mathrm{~kb}$ down & 71.8 & + & NM_020296 & Rbms1 & Other intron & 50.3 & + \\
\hline NM_008494 & Lfng & 1st intron & 71.8 & + & NM_023049 & Asb2 & $10 k-5$ kb down & 44.4 & + \\
\hline NM_027268 & Scrn1 & 1st intron & 70.2 & + & NM_010919 & Nkx2-2 & $1 \mathrm{k}-5 \mathrm{~kb}$ up & 44.4 & - \\
\hline NM_001029850 & Magi1 & Other intron & 69.4 & + & NM_011137 & Pou2f1 & 1st intron & 44.4 & + \\
\hline NM_010439 & Hmgb1 & $0-300$ b up & 66.9 & - & NM_021458 & Fzd3 & 1st intron & 42.4 & + \\
\hline NM_011319 & Sars & Other intron & 63.9 & + & NM_028778 & Nuak2 & 1st intron & 42.4 & + \\
\hline NM_010898 & Nf2 & $0-300$ b up & 63.9 & - & NM_008952 & Pipox & $0-300$ b up & 41.4 & + \\
\hline NM_011137 & Pou2f1 & 1st intron & 62.3 & + & NM_172913 & Tnrc9 & $0-5 \mathrm{~kb}$ down & 40.9 & - \\
\hline NM_011817 & Gadd45g & $0-300$ b up & 62.3 & - & NM_007434 & Akt2 & Other intron & 39.5 & - \\
\hline NM_028778 & Nuak2 & 1st intron & 57.5 & + & NM_008494 & Lfng & 1st intron & 39.5 & + \\
\hline NM_001039484 & Kcnj10 & 1st intron & 55.9 & - & NM_175274 & Ttyh3 & $10 \mathrm{k}-5 \mathrm{~kb}$ down & 39.5 & + \\
\hline NM_146057 & Dap & $0-300$ b up & 55.1 & - & NM_183144 & Inpp5a & 0ther intron & 38.5 & + \\
\hline NM_029756 & Sdccag8 & $300-1 \mathrm{~kb}$ up & 54.3 & - & NM_001025074 & Ntrk2 & Other intron & 38.0 & + \\
\hline NM_201610 & Neil2 & $0-5 \mathrm{~kb}$ down & 54.3 & + & NM_027216 & Slc39a11 & Other intron & 38.0 & + \\
\hline NM_010308 & Gnao1 & Other intron & 54.3 & + & NM_028263 & Fgfbp3 & $10 \mathrm{k}-5 \mathrm{~kb}$ down & 37.5 & - \\
\hline NM_010191 & Fdft1 & $0-300$ b up & 54.3 & + & NM_201357 & Tssc1 & Other intron & 37.0 & + \\
\hline NM_008997 & Rab11b & $0-300$ b up & 53.5 & - & NM_027268 & Scrn1 & 1st intron & 36.5 & + \\
\hline NM_201357 & Tssc1 & 0ther intron & 52.7 & + & NM_010097 & Sparcl1 & 1st intron & 36.0 & + \\
\hline NM_025980 & Nrarp & $0-300$ b up & 52.7 & - & NM_011936 & Fto & 1st intron & 35.5 & - \\
\hline NM_013827 & Mtf2 & $0-300$ b up & 51.9 & - & NM_172544 & Nrxn3 & Other intron & 35.5 & + \\
\hline NM_174988 & $\mathrm{Cdh} 22$ & Other intron & 51.1 & - & NM_007458 & Ap2a1 & $10 \mathrm{k}-5 \mathrm{~kb}$ down & 34.5 & - \\
\hline NM_008629 & Msi1 & Other intron & 50.3 & - & ВС057127 & Fuz & $10 \mathrm{k}-5 \mathrm{~kb}$ up & 34.5 & - \\
\hline AK163963 & Gm1967 & 1st intron & 50.3 & + & NM_029365 & Med25 & $300-1 \mathrm{~kb}$ up & 34.5 & - \\
\hline NM_010913 & Nfya & $0-300$ b up & 49.5 & - & NM_054043 & Msi2 & Other intron & 34.5 & + \\
\hline NM_009694 & Apobec2 & $10 \mathrm{k}-5 \mathrm{~kb}$ down & 49.5 & - & NM_009846 & $\mathrm{Cd} 24 \mathrm{a}$ & 1st intron & 33.5 & + \\
\hline NM_207219 & Al314976 & $0-300$ b up & 49.5 & - & NM_016968 & Olig1 & $10 k-5$ kb up & 33.5 & - \\
\hline NM_178642 & Tmem16a & 0ther intron & 48.7 & + & NM_011319 & Sars & Other intron & 33.5 & + \\
\hline NM_198247 & Sertad4 & $10 \mathrm{k}-5 \mathrm{~kb}$ down & 48.7 & - & NM_178765 & 5730410E15Rik & Other intron & 33.0 & - \\
\hline NM_020296 & Rbms1 & Other intron & 48.7 & + & NM_010419 & Hes5 & $0-300$ b up & 33.0 & + \\
\hline NM_054043 & Msi2 & Other intron & 48.7 & + & NM_027032 & Pacrg & Other intron & 33.0 & + \\
\hline NM_011544 & Tff12 & Other intron & 47.9 & - & NM_172990 & Pank4 & $1 \mathrm{k}-5 \mathrm{~kb}$ up & 33.0 & + \\
\hline NM_010952 & 0az2 & $0-300$ b up & 47.9 & - & NM_027504 & Prdm16 & Other intron & 33.0 & - \\
\hline NM_026201 & Ccar1 & $0-300$ b up & 47.9 & - & NM_027539 & Dcamkl2 & 0ther intron & 32.6 & - \\
\hline NM_181585 & Pik3r3 & $300-1 \mathrm{~kb}$ up & 46.3 & - & NM_177618 & ВС030477 & 1st intron & 31.6 & + \\
\hline NM_026272 & Narf & $0-300$ b up & 46.3 & - & NM_172522 & Megf11 & 0ther intron & 31.6 & + \\
\hline NM_016701 & Nes & Other intron & 43.1 & + & NM_016701 & Nes & 0ther intron & 27.6 & + \\
\hline
\end{tabular}

The 284 sites from the Sox21 ChIP sequencing data and the 301 sites from the Sox2 ChIP sequencing data were determined as described in Materials and Methods. Genes with these binding sites near their TSSs were annotated as bound genes and are shown here in decreasing order of maximum $p$ values $\left(-\log _{10} p\right)$. The top 40 genes and the Nestin gene are listed. The peak location is displayed relative to the genomic distance from the TSS. up, Upstream of TSS; down, downstream of TSS. If the peak resided within a coding region, the location is described as the 5' UTR or introns. A plus sign in the 0verlap column indicates that the gene in question was listed as both a Sox21 and Sox 2 candidate target gene. The Nestin gene, which is expressed in NS/PCS, was bound by Sox2, consistent with a previous report showing that Sox2 binds to the second intron of the Nestin gene to modulate its expression (Zimmerman et al., 1994).

genesis, we examined whether Hes 5 overexpression was capable of canceling the neurogenic function of Sox 21. Simultaneous expression of both proteins in NS/PCs in vivo was achieved by transducing cells with a construct containing Sox 21 and Hes5 fused to a self-cleaving $2 \mathrm{~A}$ peptide. Interestingly, compared with cells overexpressing Sox21 alone, the proportion of NeuNpositive cells was dramatically reduced in cells overexpressing both Sox21 and Hes5 (Fig. 6E,F). This result clearly indicates an antagonistic effect of Hes5 on Sox 21 in the context of adult neurogenesis in the DG.

\section{Discussion}

The present study provides evidence for a Sox21-mediated novel regulatory mechanism during adult neurogenesis in the DG. Sox 21 represses the transcription of Hes 5 by binding to the DRE in the Hes 5 gene. Hes 5 repression by Sox 21 is a vital event directing the neuronal differentiation of NS/PCs.

\section{Essential role of Sox 21 during adult neurogenesis}

In the present study, we demonstrated that Sox 21 was expressed in type 1 stem-like cells and type 2a progenitors in the adult hippocampal DG (Fig. 2). Our in vivo analysis of Sox $21^{-/-}$mice revealed the expansion of type 2 a cells and the reduction of type $2 \mathrm{~b}$ cells (Fig. 4, 5). Moreover, we found that exogenous expression of Sox 21 promoted neuronal differentiation in the DG. These findings lead to the conclusion that the major function of Sox 21 is to drive the progression of progenitors toward differentiation, and that Sox 21 exerts this function principally when NS/ PCs are in the type 2 a cell stage in the adult hippocampal DG.

Previous studies indicate that Ascl1 (Pleasure et al., 2000) and Ngn2 (Galichet et al., 2008) are factors that determine differentiation in embryonic DG morphogenesis. The neurogenic functions of Ascl1 and Ngn2 are predicted to be conserved in adult hippocampal neurogenesis due to their continued expression in the adult DG (Kim et al., 2007; Ozen et al., 2007). However, this 
A

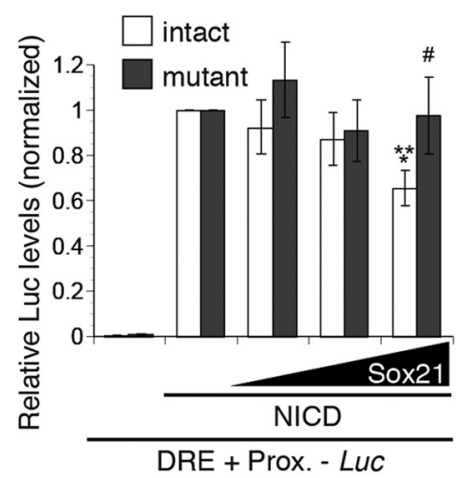

B

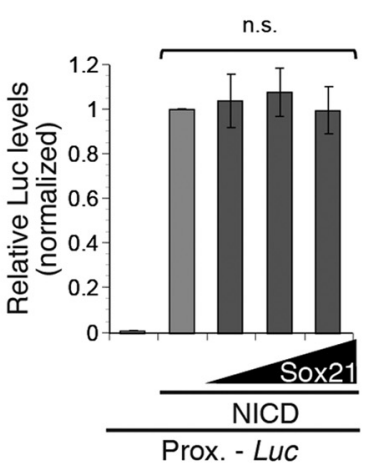

C

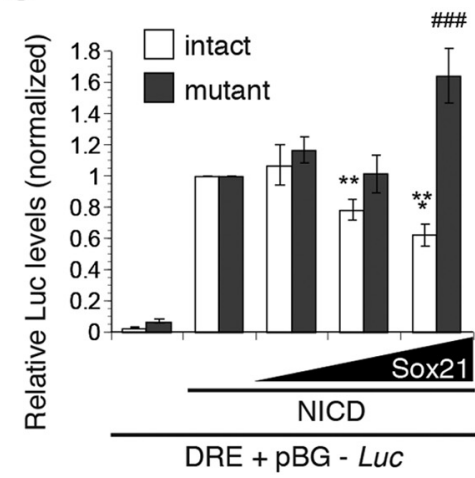

D

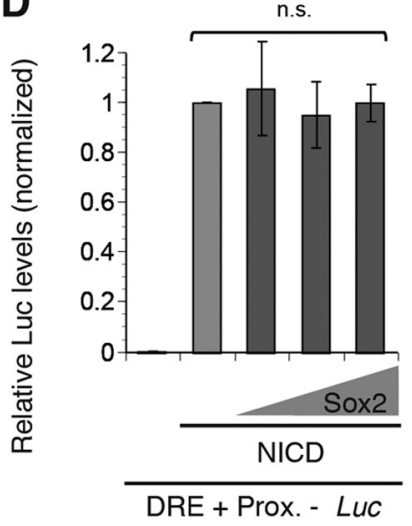

E

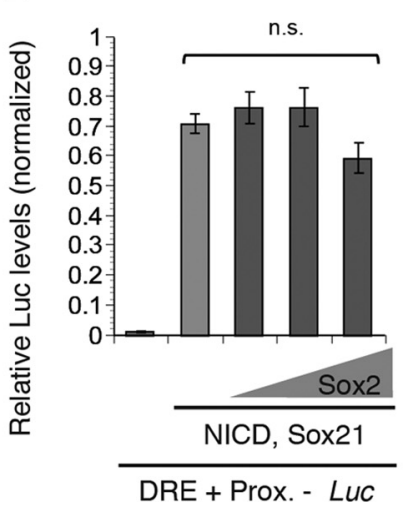

Figure 8. Repression of Hes5 gene promoter activity by Sox21 but not Sox2. AHP cells were transfected with Hes5 promoter-luciferase reporter constructs (1 $\mu$ g per well) with or without expression vectors for NICD, Sox21, and/or Sox2. The histogram shows the ratio of firefly luciferase to that in cells transfected with the reporter construct and the NICD-expressing vector. The transfection efficiency was normalized to Renilla luciferase activity. $\boldsymbol{A}, \boldsymbol{B}$, The Hes5 DRE and proximal promoter $(-2767$ to +73 bp from TSS) and the proximal promoter alone ( -687 to +73 bp) were used as reporter constructs in $\boldsymbol{A}$ and $\boldsymbol{B}$, respectively. The open columns in $\boldsymbol{A}$ show the relative luciferase activities using the intact reporter construct, and the closed columns reflect the construct mutagenized at the Sox-binding sequence of the DRE (ACAAAGG to AagcttG). NICD-expression vector, $0.1 \mu$ g per well; Sox 21-expression vector, $0.0001,0.001$, and $0.01 \mu \mathrm{g}$ per well. $C$, The Hes 5 DRE $(-2676$ to $-2244 \mathrm{bp})$ combined with the $\beta$-globin minimal promoter was used. The open columns indicate the intact reporter construct, and the closed columns indicate the mutagenized construct as shown in $\boldsymbol{A}$. NICD, $1 \mu \mathrm{g}$ per well; Sox21, 0.0001, 0.001, and $0.01 \mu \mathrm{g}$ per well. D, E, The Hes5 DRE and proximal promoter were used. D, NICD, $0.1 \mu \mathrm{g}$ per well, Sox2, 0.0001, 0.001, and $0.01 \mu \mathrm{g}$ per well. $\boldsymbol{E}$, NICD, $0.1 \mu \mathrm{g}$ per well; Sox21, $0.01 \mu \mathrm{g}$ per well; Sox2, $0.0001,0.001$, and $0.01 \mu \mathrm{g}$ per well. Data represent the mean \pm SE. Statistical significance versus the intact promoter was assessed by two-sided $t$ test $(n>4) .{ }^{* *} p<0.01,{ }^{* * *} p<0.001$ versus NICD alone; ${ }^{\#} p<0.05 ;{ }^{\# \# \#} p<0.001$ versus intact promoter. n.S., No significance versus NICD and Sox21 alone according to the two-sided $t$ test $(n>4)$. Prox., Proximal promoter.
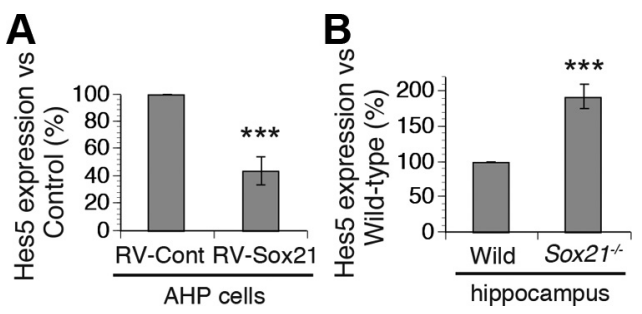

Figure 9. Repressive role of Sox21 in Hes5 expression in vitro and in vivo. $\boldsymbol{A}$, In vitro analysis of Hes5 expression level confirmed by RT-qPCR. AHP cells infected with a Sox21-expressing retrovirus (RV-Sox21) expressed Hes5 at a lower level than controls (RV-Cont). ${ }^{* *} p<0.001$ (two-sided $t$ test). $n=5$. $\boldsymbol{B}$, Endogenous Hes5 expression level in 6-week-old wild-type and Sox $21^{-/-}$hippocampus determined using RT-qPCR. ${ }^{* * *} p<0.001$ versus wild-type mice (two-sided $t$ test). $n=4$. Data represent the mean $\pm S E$.

hypothesis is controversial because Ascll overexpression in adult DG was reported to redirect the fate of NS/PCs into oligodendrocyte lineage (Jessberger et al., 2008). Here, we present the first evidence from both in vivo loss- and gain-of-function experiments showing that a single transcription factor, Sox21, can govern the transition of progenitors into neuronal differentiation stages in the adult hippocampus.
The generation of new neurons was significantly abrogated in So $21^{-1-}$ mice; however, hippocampal neurogenesis was not completely abolished (Fig. 4). This observation may be attributable to the existence of some NS/PCs that pursue their neurogenic steps in a Sox 21 -independent manner, judging from the existence of Sox21-negative NS/PCs in the DG (Fig. 2). Moreover, other factors in addition to Sox 21 may play a role in Hes5 gene repression. For example, the Cdc42-mammalian target of rapamycin pathway (Endo et al., 2009) and Fezf proteins (Shimizu et al., 2010) control the neuronal differentiation of NS/PCs by repressing the Hes 5 gene. These signaling pathways may have compensated for the loss of Sox 21 by repressing Hes 5 transcription.

\section{Sox 21-mediated regulation of Hes 5 expression in NS/PCs} In the adult mammalian brain, Notch signaling is involved in the maintenance of NS/PCs, and the conditional knock-out of CSL forces NS/PCs to differentiate into neurons (Imayoshi et al., 2010). Likewise, the abrogation of Notch signaling leads to the precocious transition of NS/PCs into neurons in the adult hippocampal DG, and overexpression of NICD induces the expansion of the highly proliferative NS/PC population (Breunig et al., 2007). Hes5 is a canonical downstream effector of Notch signal- 
A
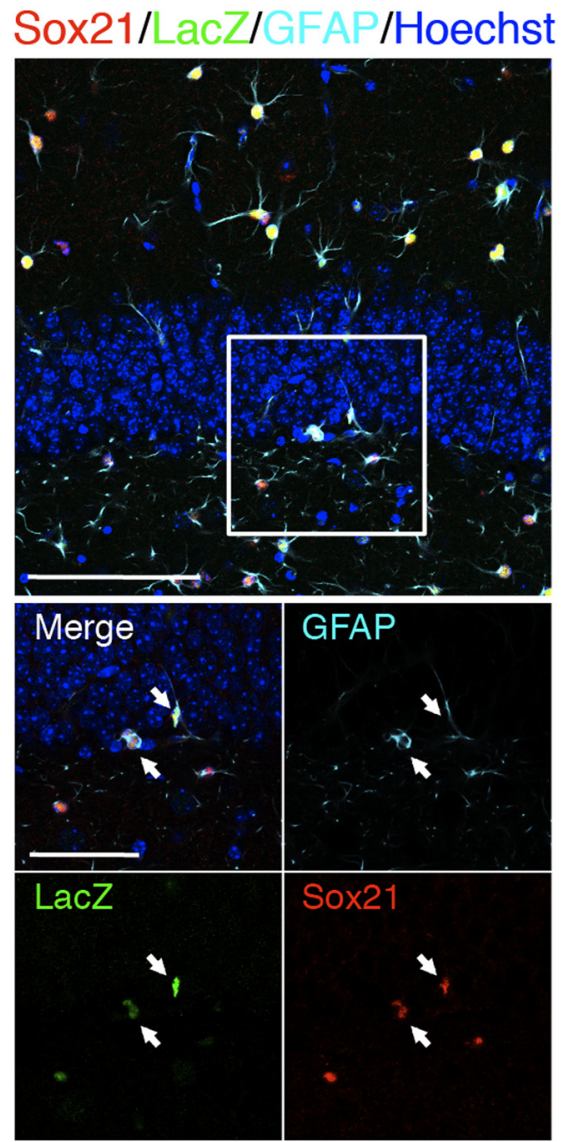

B
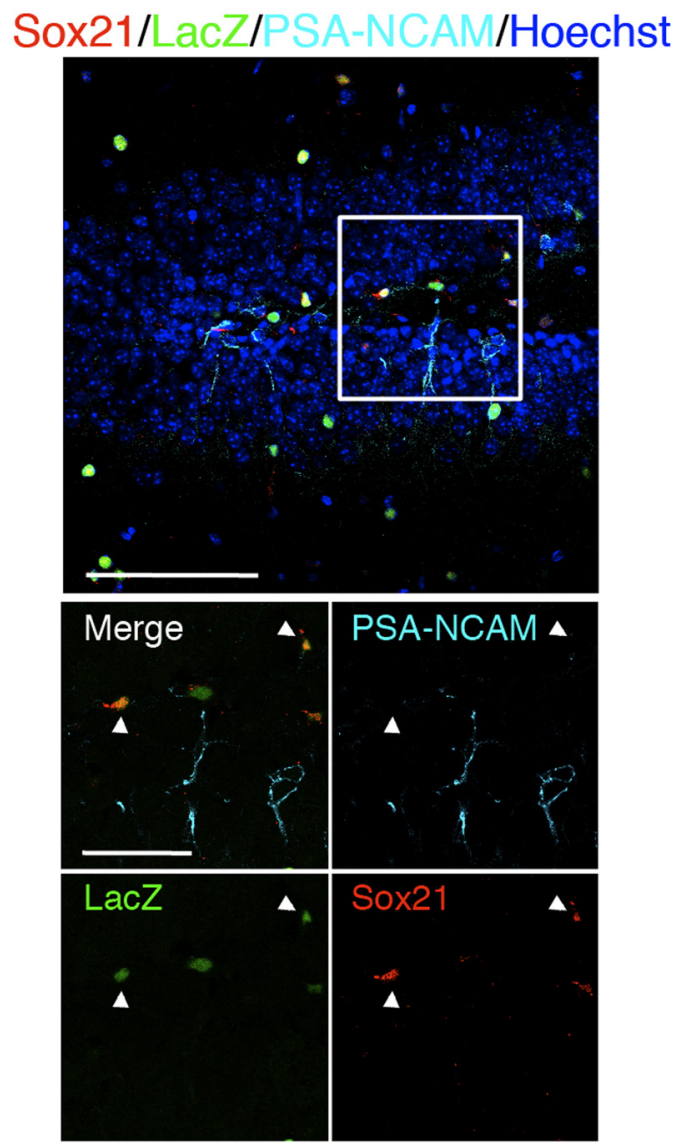

Figure 10. Colocalization of Sox21 and Hes5 in a small portion of NS/PCs, but not in immature neurons. $\boldsymbol{A}, \boldsymbol{B}$, Triple immunohistochemical analyses of Hes5 expression in the adult DG using Hes5-NLSlacZ knock-in mice. A, Triple staining for Sox21, Hes5 (lacZ), and the NS/PC marker GFAP. Arrows indicate Sox21+/Hes5+/GFAP + cells. B, Triple staining for Sox21, Hes5, and the immature neuronal marker PSA-NCAM. Arrowheads indicate Sox21+/Hes5+/PSA-NCAM cells. Scale bars: top row, $100 \mu \mathrm{m}$; magnified, $50 \mu \mathrm{m}$.

A

RV- RVshControl shHes5

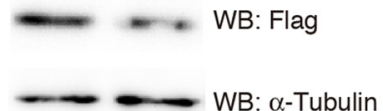

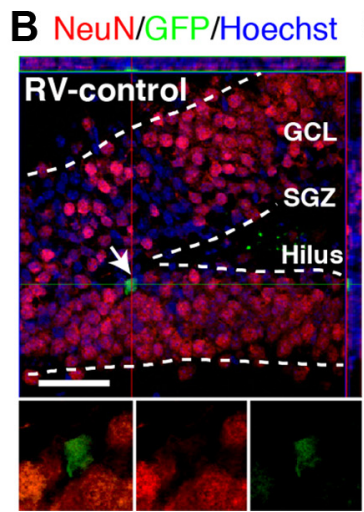

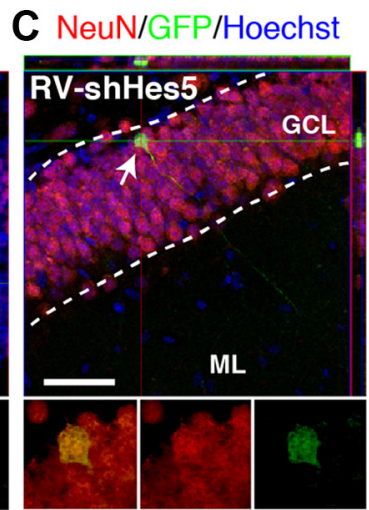

D

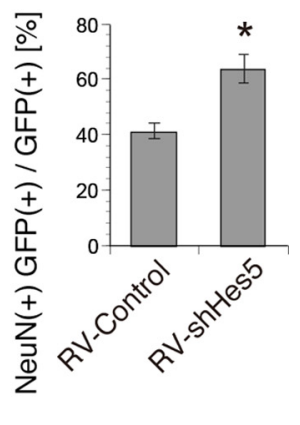

Figure 11. Suppression of Hes 5 expression promoted neuronal differentiation in the adult DG. A, Western blotting to validate Hes 5 shRNA. NIH3T3 cells stably expressing FLAG-Hes 5 were infected with a retrovirus encoding control shRNA (RV-shControl) or Hes5 shRNA (RV-shHes5) for $7 \mathrm{~d}$. $\alpha$-Tubulin expression was used as an internal control. $\boldsymbol{B}, \mathrm{C}$, Micrographs of retrovirus-labeled GFP+ newborn cells (arrows) at $21 \mathrm{~d}$ after retrovirus infusion into the DG of 6-week-old wild-type mice. Control shRNA-overexpressing $(\boldsymbol{B})$ and Hes5 shRNA-overexpressing ( $\boldsymbol{C}$ ) groups are shown. The GFP-positive infected cells in C demonstrated NeuN expression. D, Quantification of the neuronal differentiation of newborn cells at $21 \mathrm{~d}$ after retrovirus injection. Newborn neurons were identified by the presence of NeuN expression, and the data are expressed as the proportion of NeuN + cells among the total GFP + infected cell population in the SGZ and GCL. Data represent the mean \pm SE. ${ }^{*} p<0.05$ (two-sided $t$ test). $n=4$. Scale bars: $50 \mu \mathrm{m}$.

ing (Ohtsuka et al., 1999), which inhibits neuronal differentiation by repressing proneural genes such as Mash1, Math, and Neurogenin (Kageyama et al., 2005), and is believed to be involved in the maintenance of the undifferentiated status of NS/PCs in the adult DG (Lugert et al., 2010). Hes5 expression is mainly activated by binding of the CSL transcription factor to the Hes5 proximal promoter (Takebayashi et al., 1995; Ong et al., 2006). However, because of the existence of Sox-binding elements in the Hes5 promoter, Sox family proteins are also predicted to control Hes 5 expression (Takanaga et al., 2009). To the best of our knowledge, the present study is the first to show that Sox 21 binds to the Hes5 gene and represses its transcription (Figs. 7-9). Furthermore, the 
repressive effect of Sox 21 was blocked by introducing a mutation into the Sox-binding site in a novel distal Hes5 regulatory element (Fig. 8), suggesting that Sox21 may negatively regulate Notchdownstream signaling via a Notch/CSL-independent pathway. Furthermore, we showed that the Sox 21 -mediated repression of Hes5 was sufficient to allow the neuronal differentiation of NS/ PCs in the adult DG (Fig. 6). Interestingly, the Hes5 proximal promoter was not responsible for the Sox 21 -mediated regulation of Hes 5 transcription, implying that the DRE plays a notable role in the progression of adult neurogenesis.

Although our motif analyses revealed the possibility that Sox 21 and Sox 2 proteins recognize cis-elements in a similar way (Fig. $7 B$ ), only 97 genomic loci (34.5\% of the loci bound by Sox 21 and $32.2 \%$ of the loci bound by Sox 2 ) turned out to be bound by both Sox 21 and Sox2 (Fig. 7A). Furthermore, regulation of Hes5 gene expression differed between these Sox proteins: Sox21, but not Sox2, bound to the Hes5 DRE. The fact that Sox 2 did not bind to the Hes5 DRE may explain why Sox 2 did not antagonize Sox 1 in the regulation of Hes 5 gene expression. To our knowledge, this is the first example of competition between SoxB1 and SoxB2 proteins. To better understand this phenomenon, it would be interesting to identify the transcriptional factor complex recruited by Sox 21 . The presence of the conserved POU3f2 (Brn2) octamer sequence close to the Sox motif in the Hes5 DRE (Fig. $7 D)$ implies that Brn2 may participate in regulating Hes5 expression through Sox 21 . The fact that Sox 2 and Brn2 synergistically regulate Nestin gene expression by binding to adjacent Sox- and POU-binding motifs, respectively (Zimmerman et al., 1994; Tanaka et al., 2004), supports the idea of interplay between Sox protein and Brn2.

From the finding that simultaneous overexpression of Hes5 and Sox 21 in the adult DG blocked the neuronal differentiation induced by retrovirus-delivered Sox 21 (Fig. 6E,F), we concluded that the Hes 5 gene is a functional downstream target of Sox 21 . However, the involvement of other possible target genes in the progression of Sox 21 -induced neuronal differentiation cannot be excluded. ChIP sequencing results identified other candidate genes likely related to brain development. For example, Sox 21 seems to bind to the intronic region of the Msi1 $\left(-\log _{10} p=50.3\right)$ and Msi2 genes $\left(-\log _{10} p=48.7\right)$ (Table 1$)$. Musashi-1 is highly expressed in NS/PCs in the mammalian CNS (Sakakibara et al., 1996; Tonchev et al., 2005) and is thought to contribute to NS/PC self-renewal (Okano et al., 2005) and hippocampal adult neurogenesis (Kempermann et al., 2006). It would be of interest to examine the role of Sox21 in the transcriptional regulation of Msil.

Furthermore, the ChIP sequencing results revealed that Sox 21 may bind to genes related to Notch signaling: for example, the Lfng gene, which encodes Lunatic fringe protein $\left(-\log _{10} p=\right.$ 71.8), and the Pou2f1 (Oct1) gene $\left(-\log _{10} p=62.3\right)$ (Table 1$)$. Lfng expression is restricted to the ventricular zone of developing cortical walls in mice (Ishii et al., 2000) and potentiates Dll-1mediated Notch signaling to maintain the proliferation of NS/ PCs (Kato et al., 2010). Pou2f1 expression is reportedly induced by NICD and is required for radial glia formation in the Xenopus hindbrain (Kiyota et al., 2008). Intriguingly, Sox 21 tends to enhance the expression of $L f n g$ and suppress Pou $2 f 1$ expression in AHP cells (our unpublished results). Although the detailed expression profiles of $L f n g$ and Pou $2 f 1$ in the adult brain, and their roles in regulating hippocampal adult neurogenesis, remain unknown, Sox 21 may exert its neurogenic function by determining the optimal level of Notch signaling transduction by controlling these Notch-related factors.
This study demonstrated that Sox 21 regulates adult neurogenesis in vivo and contributes to the generation of new neurons. Recently, increasing evidence has revealed a profound relationship between adult neurogenesis and learning (Shors et al., 2001), depression (Malberg et al., 2000), and some neurodegenerative diseases, including Huntington's disease (Curtis et al., 2003), Parkinson's disease (Winner et al., 2004), and Alzheimer's disease (Wen et al., 2004; Donovan et al., 2006). It is therefore of great value to focus on the involvement of Sox 21 in the physiology of brain function as well as the pathophysiology of such diseases. Identifying targets other than Hes 5 using our genomewide ChIP sequencing information should help to increase the current understanding of the gene network that controls adult neurogenesis.

\section{References}

Akazawa C, Sasai Y, Nakanishi S, Kageyama R (1992) Molecular characterization of a rat negative regulator with a basic helix-loop-helix structure predominantly expressed in the developing nervous system. J Biol Chem 267:21879-21885.

Bailey TL, Elkan C (1994) Fitting a mixture model by expectation maximization to discover motifs in biopolymers. Proc Int Conf Intell Syst Mol Biol 2:28-36.

Bani-Yaghoub M, Tremblay RG, Lei JX, Zhang D, Zurakowski B, Sandhu JK, Smith B, Ribecco-Lutkiewicz M, Kennedy J, Walker PR, Sikorska M (2006) Role of Sox2 in the development of the mouse neocortex. Dev Biol 295:52-66.

Breunig JJ, Silbereis J, Vaccarino FM, Sestan N, Rakic P (2007) Notch regulates cell fate and dendrite morphology of newborn neurons in the postnatal dentate gyrus. Proc Natl Acad Sci U S A 104:20558-20563.

Curtis MA, Penney EB, Pearson AG, van Roon-Mom WM, Butterworth NJ, Dragunow M, Connor B, Faull RL (2003) Increased cell proliferation and neurogenesis in the adult human Huntington's disease brain. Proc Natl Acad Sci U S A 100:9023-9027.

Donovan MH, Yazdani U, Norris RD, Games D, German DC, Eisch AJ (2006) Decreased adult hippocampal neurogenesis in the PDAPP mouse model of Alzheimer's disease. J Comp Neurol 495:70-83.

Duan X, Kang E, Liu CY, Ming GL, Song H (2008) Development of neural stem cell in the adult brain. Curr Opin Neurobiol 18:108-115.

Endo M, Antonyak MA, Cerione RA (2009) Cdc42-mTOR signaling pathway controls Hes5 and Pax6 expression in retinoic acid-dependent neural differentiation. J Biol Chem 284:5107-5118.

Gage FH (2000) Mammalian neural stem cells. Science 287:1433-1438.

Galichet C, Guillemot F, Parras CM (2008) Neurogenin 2 has an essential role in development of the dentate gyrus. Development 135:2031-2041.

Hodge RD, Kowalczyk TD, Wolf SA, Encinas JM, Rippey C, Enikolopov G, Kempermann G, Hevner RF (2008) Intermediate progenitors in adult hippocampal neurogenesis: Tbr2 expression and coordinate regulation of neuronal output. J Neurosci 28:3707-3717.

Imaizumi Y, Sakaguchi M, Morishita T, Ito M, Poirier F, Sawamoto K, Okano H (2011) Galectin-1 is expressed in early-type neural progenitor cells and down-regulates neurogenesis in the adult hippocampus. Mol Brain 4:7.

Imayoshi I, Sakamoto M, Yamaguchi M, Mori K, Kageyama R (2010) Essential roles of Notch signaling in maintenance of neural stem cells in developing and adult brains. J Neurosci 30:3489-3498.

Ishii Y, Nakamura S, Osumi N (2000) Demarcation of early mammalian cortical development by differential expression of fringe genes. Brain Res Dev Brain Res 119:307-320.

Jessberger S, Toni N, Clemenson GD Jr, Ray J, Gage FH (2008) Directed differentiation of hippocampal stem/progenitor cells in the adult brain. Nat Neurosci 11:888-893.

Kageyama R, Ohtsuka T, Hatakeyama J, Ohsawa R (2005) Roles of bHLH genes in neural stem cell differentiation. Exp Cell Res 306:343-348.

Kan L, Israsena N, Zhang Z, Hu M, Zhao LR, Jalali A, Sahni V, Kessler JA (2004) Sox1 acts through multiple independent pathways to promote neurogenesis. Dev Biol 269:580-594.

Kaneshiro K, Tsutsumi S, Tsuji S, Shirahige K, Aburatani H (2007) An integrated map of p53-binding sites and histone modification in the human ENCODE regions. Genomics 89:178-188.

Kato TM, Kawaguchi A, Kosodo Y, Niwa H, Matsuzaki F (2010) Lunatic 
fringe potentiates Notch signaling in the developing brain. Mol Cell Neurosci 45:12-25.

Kel AE, Gössling E, Reuter I, Cheremushkin E, Kel-Margoulis OV, Wingender E (2003) MATCH: A tool for searching transcription factor binding sites in DNA sequences. Nucleic Acids Res 31:3576-3579.

Kempermann G, Jessberger S, Steiner B, Kronenberg G (2004) Milestones of neuronal development in the adult hippocampus. Trends Neurosci 27:447-452.

Kempermann G, Chesler EJ, Lu L, Williams RW, Gage FH (2006) Natural variation and genetic covariance in adult hippocampal neurogenesis. Proc Natl Acad Sci U S A 103:780-785.

Kim EJ, Leung CT, Reed RR, Johnson JE (2007) In vivo analysis of Ascl1 defined progenitors reveals distinct developmental dynamics during adult neurogenesis and gliogenesis. J Neurosci 27:12764-12774.

Kiso M, Tanaka S, Saba R, Matsuda S, Shimizu A, Ohyama M, Okano HJ, Shiroishi T, Okano H, Saga Y (2009) The disruption of Sox21-mediated hair shaft cuticle differentiation causes cyclic alopecia in mice. Proc Natl Acad Sci U S A 106:9292-9297.

Kitamura T, Koshino Y, Shibata F, Oki T, Nakajima H, Nosaka T, Kumagai H (2003) Retrovirus-mediated gene transfer and expression cloning: powerful tools in functional genomics. Exp Hematol 31:1007-1014.

Kiyota T, Kato A, Altmann CR, Kato Y (2008) The POU homeobox protein Oct-1 regulates radial glia formation downstream of Notch signaling. Dev Biol 315:579-592.

Lathia JD, Mattson MP, Cheng A (2008) Notch: from neural development to neurological disorders. J Neurochem 107:1471-1481.

Lefebvre V, Dumitriu B, Penzo-MéndezA, Han Y, Pallavi B (2007) Control of cell fate and differentiation by Sry-related high-mobility-group box (Sox) transcription factors. Int J Biochem Cell Biol 39:2195-2214.

Lewis J (1996) Neurogenic genes and vertebrate neurogenesis. Curr Opin Neurobiol 6:3-10.

Lugert S, Basak O, Knuckles P, Haussler U, Fabel K, Götz M, Haas CA, Kempermann G, Taylor V, Giachino C (2010) Quiescent and active hippocampal neural stem cells with distinct morphologies respond selectively to physiological and pathological stimuli and aging. Cell Stem Cell 6:445-456.

Malberg JE, Eisch AJ, Nestler EJ, Duman RS (2000) Chronic antidepressant treatment increases neurogenesis in adult rat hippocampus. J Neurosci 20:9104-9110.

Ohba H, Chiyoda T, Endo E, Yano M, Hayakawa Y, Sakaguchi M, Darnell RB, Okano HJ, Okano H (2004) Sox21 is a repressor of neuronal differentiation and is antagonized by YB-1. Neurosci Lett 358:157-160.

Ohtsuka T, Ishibashi M, Gradwohl G, Nakanishi S, Guillemot F, Kageyama R (1999) Hes1 and Hes5 as notch effectors in mammalian neuronal differentiation. EMBO J 18:2196-2207.

Okano H, Kawahara H, Toriya M, Nakao K, Shibata S, Imai T (2005) Function of RNA-binding protein Musashi-1 in stem cells. Exp Cell Res 306:349-356.

Ong CT, Cheng HT, Chang LW, Ohtsuka T, Kageyama R, Stormo GD, Kopan R (2006) Target selectivity of vertebrate notch proteins. Collaboration between discrete domains and CSL-binding site architecture determines activation probability. J Biol Chem 281:5106-5119.

Ozen I, Galichet C, Watts C, Parras C, Guillemot F, Raineteau O (2007) Proliferating neuronal progenitors in the postnatal hippocampus transiently express the proneural gene Ngn2. Eur J Neurosci 25:2591-2603.

Palmer TD, Takahashi J, Gage FH (1997) The adult rat hippocampus contains primordial neural stem cells. Mol Cell Neurosci 8:389-404.

Pleasure SJ, Collins AE, Lowenstein DH (2000) Unique expression patterns of cell fate molecules delineate sequential stages of dentate gyrus development. J Neurosci 20:6095-6105.

Sakaguchi M, Shingo T, Shimazaki T, Okano HJ, Shiwa M, Ishibashi S, Oguro H, Ninomiya M, Kadoya T, Horie H, Shibuya A, Mizusawa H, Poirier F,
Nakauchi H, Sawamoto K, Okano H (2006) A carbohydrate-binding protein, Galectin-1, promotes proliferation of adult neural stem cells. Proc Natl Acad Sci U S A 103:7112-7117.

Sakakibara S, Imai T, Hamaguchi K, Okabe M, Aruga J, Nakajima K, Yasutomi D, Nagata T, Kurihara Y, Uesugi S, Miyata T, Ogawa M, Mikoshiba K, Okano H (1996) Mouse-Musashi-1, a neural RNA-binding protein highly enriched in the mammalian CNS stem cell. Dev Biol 176:230-242.

Sandberg M, Källström M, Muhr J (2005) Sox21 promotes the progression of vertebrate neurogenesis. Nat Neurosci 8:995-1001.

Seri B, García-Verdugo JM, McEwen BS, Alvarez-Buylla A (2001) Astrocytes give rise to new neurons in the adult mammalian hippocampus. J Neurosci 21:7153-7160.

Shimizu T, Nakazawa M, Kani S, Bae YK, Shimizu T, Kageyama R, Hibi M (2010) Zinc finger genes Fezf1 and Fezf2 control neuronal differentiation by repressing Hes5 expression in the forebrain. Development 137:1875-1885.

Shors TJ, Miesegaes G, Beylin A, Zhao M, Rydel T, Gould E (2001) Neurogenesis in the adult is involved in the formation of trace memories. Nature 410:372-376.

Suh H, Consiglio A, Ray J, Sawai T, D’Amour KA, Gage FH (2007) In vivo fate analysis reveals the multipotent and self-renewal capacities of Sox2+ neural stem cells in the adult hippocampus. Cell Stem Cell 1:515-528.

Tada H, Ishii S, Kimura H, Hattori H, Okada Y, Suzuki N, Okano HJ (2007) Identification and evaluation of high-titer anti-Sox Group B antibody in limbic encephalitis. Inflamm Regen 27:37-44.

Takanaga H, Tsuchida-Straeten N, Nishide K, Watanabe A, Aburatani H, Kondo T (2009) Gli2 is a novel regulator of sox2 expression in telencephalic neuroepithelial cells. Stem Cells 27:165-174.

Takebayashi K, Akazawa C, Nakanishi S, Kageyama R (1995) Structure and promoter analysis of the gene encoding the mouse helix-loop-helix factor HES-5. Identification of the neural precursor cell-specific promoter element. J Biol Chem 270:1342-1349.

Tanaka S, Kamachi Y, Tanouchi A, Hamada H, Jing N, Kondoh H (2004) Interplay of SOX and POU factors in regulation of the Nestin gene in neural primordial cells. Mol Cell Biol 24:8834-8846.

Tonchev AB, Yamashima T, Sawamoto K, Okano H (2005) Enhanced proliferation of progenitor cells in the subventricular zone and limited neuronal production in the striatum and neocortex of adult macaque monkeys after global cerebral ischemia. J Neurosci Res 81:776-788.

Wakabayashi K, Okamura M, Tsutsumi S, Nishikawa NS, Tanaka T, Sakakibara I, Kitakami J, Ihara S, Hashimoto Y, Hamakubo T, Kodama T, Aburatani H, Sakai J (2009) The peroxisome proliferator-activated receptor gamma/retinoid X receptor alpha heterodimer targets the histone modification enzyme PR-Set7/Setd8 gene and regulates adipogenesis through a positive feedback loop. Mol Cell Biol 29:3544-3555.

Wang TW, Stromberg GP, Whitney JT, Brower NW, Klymkowsky MW, Parent JM (2006) Sox3 expression identifies neural progenitors in persistent neonatal and adult mouse forebrain germinative zones. J Comp Neurol 497:88-100.

Wen PH, Hof PR, Chen X, Gluck K, Austin G, Younkin SG, Younkin LH, DeGasperi R, Gama Sosa MA, Robakis NK, Haroutunian V, Elder GA (2004) The presenilin-1 familial Alzheimer disease mutant P117L impairs neurogenesis in the hippocampus of adult mice. Exp Neurol 188:224-237.

Winner B, Lie DC, Rockenstein E, Aigner R, Aigner L, Masliah E, Kuhn HG, Winkler J (2004) Human wild-type alpha-synuclein impairs neurogenesis. J Neuropathol Exp Neurol 63:1155-1166.

Zimmerman L, Parr B, Lendahl U, Cunningham M, McKay R, Gavin B, Mann J, Vassileva G, McMahon A (1994) Independent regulatory elements in the nestin gene direct transgene expression to neural stem cells or muscle precursors. Neuron 12:11-24. 